\title{
Arrow of time across five centuries of classical music
}

\author{
Alfredo González-Espinoza $\odot,{ }^{1}$ Gustavo Martínez-Mekler, ${ }^{2,3}$ and Lucas Lacasa $\odot^{4, *}$ \\ ${ }^{1}$ Department of Biology, University of Pennsylvania, Philadelphia, Pennsylvania 19104, USA \\ ${ }^{2}$ Instituto de Ciencias Físicas, Universidad Nacional Autónoma de Mexico, Cuernavaca, Mexico \\ ${ }^{3}$ Centro de Ciencias de la Complejidad, Universidad Nacional Autónoma de Mexico, Ciudad de México, Mexico \\ ${ }^{4}$ School of Mathematical Sciences, Queen Mary University of London, Mile End Road E14NS, London, United Kingdom
}

(Received 9 March 2020; accepted 26 May 2020; published 29 July 2020)

\begin{abstract}
The concept of time series irreversibility - the degree by which the statistics of signals are not invariant under time reversal—naturally appears in nonequilibrium physics in stationary systems which operate away from equilibrium and produce entropy. This concept has not been explored to date in the realm of musical scores as these are typically short sequences whose time reversibility estimation could suffer from strong finite size effects which preclude interpretability. Here we show that the so-called horizontal visibility graph method-which recently was shown to quantify such statistical property even in nonstationary signals-is a method that can estimate time reversibility of short symbolic sequences, thus unlocking the possibility of exploring such properties in the context of musical compositions. Accordingly, we analyze over 8000 musical pieces ranging from the Renaissance to the early Modern period and show that, indeed, most of them display clear signatures of time irreversibility. Since by construction stochastic processes with a linear correlation structure (such as $1 / f$ noise) are time reversible, we conclude that musical compositions have a considerably richer structure, that goes beyond the traditional properties retrieved by the power spectrum or similar approaches. We also show that musical compositions display strong signs of nonlinear correlations, that nonlinearity is correlated to irreversibility, and that these are also related to asymmetries in the abundance of musical intervals, which we associate to the narrative underpinning a musical composition. These findings provide tools for the study of musical periods and composers, as well as criteria related to music appreciation and cognition.
\end{abstract}

DOI: 10.1103/PhysRevResearch.2.033166

\section{INTRODUCTION}

The quantitative description of the structure in musical compositions has a long history of interdisciplinary research, with contributions from musical theory, information theory, and mathematics to physics. Traditional quantitative analysis of the temporal structure underlying musical pieces have mainly addressed linear correlations, such as the ones captured by spectral (Fourier) analysis, starting from the pioneering work of Voss and Clarke [1,2] and subsequently followed by a wealth of more in-depth analyses [3-13]. It is nowadays widely accepted that music presents a so-called $1 / f$ power spectrum and that this is a fingerprint of "appealing sound" [14-19]. However, recent evidence has challenged this vision, as it has been suggested that pleasantness could be also related to nonlinearities present in music compositions, a property which by definition is not captured in the power spectra [20]. These findings motivate further exploration into quantitative ways of measuring structure in music compositions that goes beyond linear theories. Amongst others, musicians and mu-

\footnotetext{
*1.1acasa@qmul.ac.uk

Published by the American Physical Society under the terms of the Creative Commons Attribution 4.0 International license. Further distribution of this work must maintain attribution to the author(s) and the published article's title, journal citation, and DOI.
}

sicologists have addressed the breakdown of continuity and temporality [21], the effect of asymmetry in melody [22], and the importance of musical irreversibility [23]. In all cases, a relationship with pleasantness has been explored.

Inspired by both statistical physics and nonlinear dynamical concepts, here we introduce statistical measures for irreversibility, nonlinearity, and asymmetry and present a description of music scores by gauging their interrelations. We primarily explore to what extent classical music manifests statistical time irreversibility and further on introduce nonlinearity and asymmetry. Simply put, a stationary signal is (statistically) time reversible if the statistical properties of the signal are invariant under time reversal [24], whereas the signal is statistically irreversible in the opposite case. For instance, white noise is a stochastic process known to be statistically reversible: if one listens to temporal white noise and subsequently to the same signal after time reversal, it is not possible to distinguish not only which is which, but also whether they sound different.

The notion of statistical time irreversibility, which has been associated with an "arrow of time," has deep relations in nonequilibrium physics with concepts such as dissipation and entropy production. For instance, the amount of entropy produced by a thermodynamic system out of equilibrium has been linked, in nonequilibrium steady states (NESS), to the extent in this system displays time irreversibility [25-27]. Interestingly, it is well known that a large family of stochastic processes - which include pink or $1 / f$ noise as a 
special case-are statistically reversible [28]. The presence or absence of statistical reversibility is a priori a well-suited concept to explore to what extent the regularities and patterns present in musical compositions go beyond linear correlation structures.

Standard methods that estimate irreversibility in (discrete) stationary signals usually require long time series sizes for an accurate estimation when the alphabet (number of different states) is large, simply because the amount of possible $m$ grams scales exponentially with the alphabet size. This is a problem expected to emerge in musical compositions, as these are seldom large, typically consisting of sequences of some hundreds of notes, and the alphabet size (e.g., the number of different notes involved in the piece) is rarely exponentially small. Here we leverage on a recently introduced approach, horizontal visibility graph irreversibility (HVG-I) [29,30], which we argue actually bridges this gap and is able to extract meaningful measures of statistical irreversibility in short sequences and applies to both stationary and nonstationary signals. We extend the method to deal with short sequences by defining a measure of HVG irreversibility-which we can link to entropy production - and a confidence index which states when a certain irreversibility value is genuine or, on the contrary, is just a finite size effect and therefore spurious. Equipped with these tools, we can then explore time series irreversibility in music. Interestingly, we find that a large amount of compositions indeed display time irreversibility and therefore can be understood as signals generated by systems which operate out of equilibrium and producing entropy. We explore in detail the relation between irreversibility, entropy production, and the presence of nonlinear temporal correlations in music, establishing that irreversibility is a key feature of musical compositions which is not related to linear information displayed by the power spectrum, and we are finally able to interpret such fingerprints in terms of musical composition.

The rest of the paper is as follows. In Sec. II we present the database, which consists of over 8000 musical compositions from 77 different composers spanning several centuries and different musical periods, from the Renaissance to the beginning of the Modern period. In Sec. III we present the theory and methods used to estimate time irreversibility and entropy production in music. In Sec. IV we present the results from these methods and complement them with additional characterization provided for nonlinearity and interval asymmetry. The former attempts to quantify the temporal correlation structure which persists in musical compositions once linear temporal correlations are removed, whereas the latter is a strongly musical-based notion. We provide a global picture by comparing the performance and relations between irreversibility, nonlinearity, and interval asymmetry, and in Sec. V we conclude.

\section{DATA SET}

We collected 8856 MIDI files of 77 different composers extracted from the Kunst der fugue MIDI data set [31]. Since each piece usually incorporates different voices (different time series, each one corresponding to a different voice), a first task was to decide which of these voices should be considered as the main voice or pitch sequence of the piece (note that the full, multivariate analysis could be done as well, and we leave that approach for a future work). To choose the pitch sequence in multivoice pieces, we used the following two-step criterion: (1) the pitch sequence must be longer than 30 notes and (2) the sequence should have the largest number of different notes from all sequences in the same piece. With this criterion we are assuming that the selected pitch sequence would have most of the relevant features in the musical piece (such as the melody or the theme with its variations). We checked that applying the criterion above was unambiguous, and only one sequence could be extracted from each piece. We processed each MIDI file by parsing it into a comma separated value (CSV) [32] format and with the aid of a Julia script [33] we extracted the pitch sequence (see left panel of Fig. 1 for an illustration). Some examples of pitch sequences extracted from individual pieces are depicted for illustration in the right panel of Fig. 1.

To have a rough idea of the characteristics of these sequences, in the right panel of Fig. 2 we display the sequence size histogram, whereas in the left panel of the same figure we depict the distribution of the number of different notes (i.e., the alphabet) per piece. As expected, typically the alphabet is too large (in principle there are 128 symbols, and in practice the average alphabet size is 30) and the sequence size too short (median 427) for standard time irreversibility methods-such as comparing the frequency of $n$-grams in the forward and backward sequence - to be applicable, hence motivating the use of graph-theoretic approaches such as the one described in the next section.

\section{TIME IRREVERSIBILITY}

\section{A. Reversibility and entropy production in stationary systems}

A stationary process is said to be time reversible if the joint probability distribution of the forward and backward process are statistically equivalent. More concretely, let $\mathscr{S}=\left(x_{1}, x_{2}, \ldots, x_{N}\right)$ be a time series of $N$ data, and denote $\mathscr{S}^{*}=\left(x_{N}, x_{N-1}, \ldots, x_{1}\right)$ the backward time series. The forward and backwards joint distributions are denoted respectively $\mathscr{P}_{F}(N):=P\left(x_{1}, x_{2}, \ldots, x_{N}\right)$ and $\mathscr{P}_{B}(N):=$ $P\left(x_{N}, x_{N-1}, \ldots, x_{1}\right)$. We say that the time series $\mathscr{S}$ is statistically time reversible if and only if $\mathscr{P}_{F}(m) \stackrel{d}{=} \mathscr{P}_{B}(m), \forall m=$ $1, \ldots, N$, where $\stackrel{d}{=}$ should be interpreted here as having two distributions which cannot be distinguished. Accordingly, statistical time reversibility is usually known as the property of a time series whose statistics remain the same when the series is flipped. Note that in practice $\mathscr{P}_{F}(m \gg 1)$ are hard to estimate, and in the event that only a single realization $\mathscr{S}$ is available, $\mathscr{P}_{F}(N)$ cannot be estimated at all. In those cases it is customary to estimate $\mathscr{P}_{F}(m)$ and $\mathscr{P}_{B}(m)$ for $2 \leqslant m \ll N$, since a sufficient condition for rejecting time reversibility is to reject indistinguishability for small $m$.

Gaussian linear processes such as white noise or colored noise or conservative chaotic processes such as Hamiltonian chaos are statistically time reversible, and related to processes in thermodynamic equilibrium in statistical physics. Nonlinear stochastic processes or dissipative chaotic processes, on the other hand, are generally found to be irreversible 
(a)

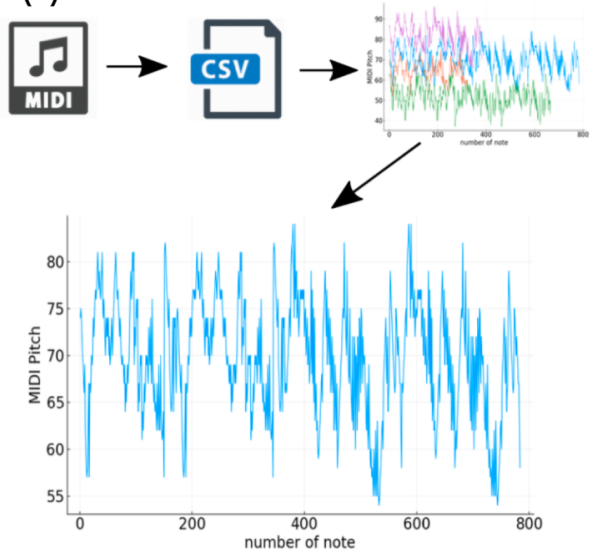

(b)

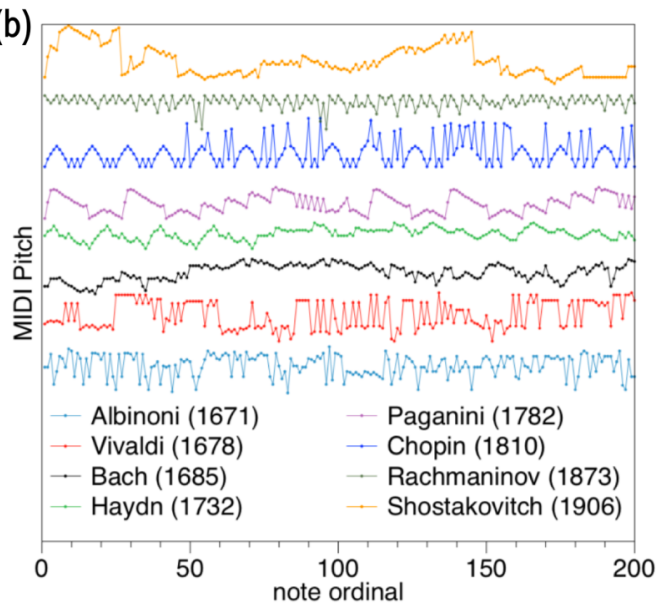

FIG. 1. Data extraction protocol. [Panel (a)] Scheme for the process of extracting one pitch sequence from each MIDI file. [Panel (b)] Samples of pieces from different authors (note that the $y$ axis has been shifted for illustration purposes).

[28], and are associated to processes that operate away from equilibrium in a thermodynamic sense.

There are various possible approaches to quantify the degree of irreversibility, starting from the obvious choice of comparing the $m$-gram statistics in $\mathscr{S}$ and $\mathscr{S}^{*}$ to more exotic approaches $[28,29,34]$. A notable result dictates that when the signal $x(t)=x_{t}$ is generated by an underlying thermodynamic system, then the amount of time irreversibility of a (infinitely long, i.e., $N \rightarrow \infty$ ) trajectory $\mathscr{S}$ is related to the amount of entropy that the underlying thermodynamic system is producing [26]. In particular, in the event that all active (i.e., out of equilibrium) degrees of freedom are characterized in the phase-space variable $x$, then the steady-state rate of entropy production $\sigma_{\text {tot }}$ is related to the time irreversibility of $\mathscr{S}$ via

$$
\sigma_{\text {tot }}=k_{B} \lim _{m \rightarrow \infty} \frac{1}{m} \operatorname{KLD}\left[\mathscr{P}_{F}(m) \| \mathscr{P}_{B}(m)\right],
$$

where $k_{B}$ is the Boltzmann constant and $\operatorname{KLD}(\cdot \| \cdot)$ is the Kullback-Leibler divergence. Note that, for any distributions $Q$ and $R, \operatorname{KLD}(Q \| R)$ defined by

$$
\operatorname{KLD}(Q \| R)=\sum_{x} Q(x) \ln [Q(x) / R(x)]
$$

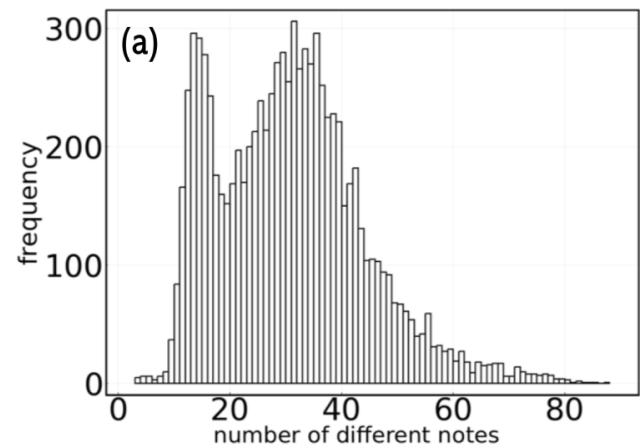

quantifies their distinguishability since $\operatorname{KLD}(Q \| R)=0$ if and only if $Q$ and $R$ are identical, and is positive otherwise. Note also that when the observable $x$ does not fully incorporate all active degrees of freedom, the right hand side in Eq. (1) is only a lower bound of the true entropy production rate. Moreover, in practice one usually cannot estimate the full hierarchy of $m$-grams, so any partial result $(m<\infty)$ again provides a lower bound to $\sigma_{\text {tot }}$. Also, when $x$ is defined on a continuous support, it is customary to symbolize it. In the case when $x$ is intrinsically discrete $x_{i} \in \mathscr{V}=\left\{v_{1}, \ldots, v_{|\mathscr{V}|}\right\}$ then a proper estimate of the right hand side (RHS) in Eq. (1) can only be attained when the sequence size $N \gg|\mathscr{V}|$ (exponentially larger): this is needed for collecting sufficient statistics on each $m$-gram. As we will show below, this latter observation is crucial in our context, as the musical compositions we consider here are relatively short symbolic sequences $(N$ ranges from a few hundred to a few thousand points), while the number of symbols (notes) present in each musical piece is typically of the same order of magnitude as $N$, thus making the direct empirical estimation of Eq. (1) ineffective.

In what follows we introduce an alternative, graphtheoretic method [29], which we show circumvents these issues.

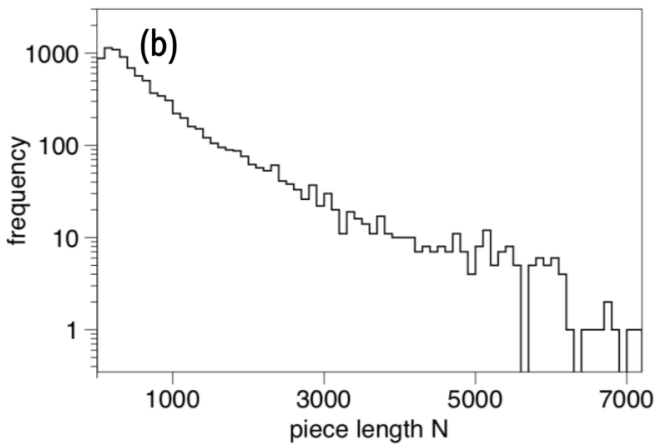

FIG. 2. Alphabet and sequence size. [Panel (a)] Histogram of the alphabet (number of different notes) for all the pieces analyzed (median $=30$ and kurtosis $=0.6$ ). [Panel (b)] Semilog histogram describing the amount of pieces that have a certain size $N$ (in number of notes). The majority of pieces have less than $10^{3}$ time steps (median $=456$ and kurtosis $=23$ ). The smallest piece has $N=35$ data points, whereas the largest has $N=15795$ data points. 


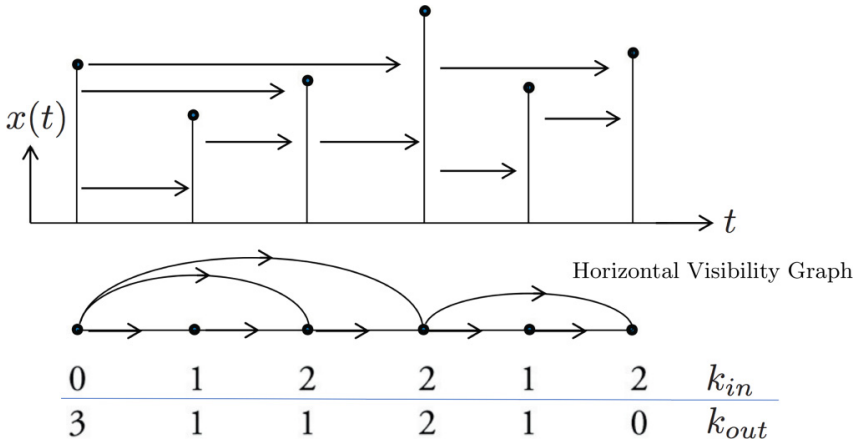

FIG. 3. Sample time series of $N=6$ data and its associated horizontal visibility graph (HVG) (see [29] for details). By assigning a temporal arrow to each of the links, the degree sequence splits into an in degree and an out degree sequence, such that flipping the time series (time reversal operation) is equivalent to interchanging the in and out degree sequences. Assessing time reversibility in the time series reduces in this context to spot differences in the statistics of the in and out degree sequences, such as comparing the in and out degree distributions.

\section{B. HVG irreversibility}

A time series of $N$ points can be transformed into a socalled horizontal visibility graph (HVG) of $N$ nodes via the so-called horizontal visibility algorithm $[35,36]$. This is a nonparametric method that enables the characterization of time series and their underlying dynamics using combinatorics and graph theory.

Definition III..1. Let $\mathscr{S}=\left\{x_{1}, \ldots, x_{N}\right\}, x_{i} \in \mathbb{R}$ be a realvalued scalar sequence of $N$ data. Its horizontal visibility graph $\operatorname{HVG}(\mathscr{S})$ is defined as an undirected graph of $N$ vertices, where each vertex $i \in\{1,2, \ldots, N\}$ is labeled in correspondence with the ordered datum $x_{i}$. Hence $x_{1}$ is related to vertex $i=1, x_{2}$ to vertex $i=2$, and so on. Then, two vertices $i, j$ (assume $i<j$ without loss of generality) share an edge if and only if $x_{k}<\inf \left(x_{i}, x_{j}\right), \forall k: i<k<j$.

HVG implements an ordering criterion which can be visualized in Fig. 3 (see [35] for a convexity criterion that generates "natural" visibility graphs instead). Visibility and horizontal visibility graphs were introduced in the context of time series analysis with the aims of using the tools of graph theory and network science [37] to describe the structure of time series and their underlying dynamics from a combinatorial perspective (for other proposals for graph-theoretical time series analysis, see [38,39]).

Among others, the concept of time series irreversibility has been recently explored within the context of visibility graphs $[29,30,40]$. In a nutshell, if the HVGs of $\mathscr{S}$ and $\mathscr{S}^{*}$ have the same properties, then $\mathscr{S}$ is said to be HVG reversible, and the concept has been shown to be applicable both in stationary and nonstationary processes [30]. How is HVG reversibility checked in practice? Since each node $i=1, \ldots, N$ in the HVG is associated to a datum $x_{i}, i=1, \ldots, N$ in the graph, there is a natural node ordering associated to the arrow of time. Such ordering is therefore inherited by the degree sequence, which has a natural representation $\mathbf{k}=\left(k_{1}, \ldots, k_{N}\right)$, where $k_{i}$ is the degree of node $i$ (i.e., the number of links adjacent to node $i$ ). Now, while the HVG is initially an undirected graph, it can be converted into a directed one by assigning a direction to each link in the HVG such that if $i<j$, then the link is $i \rightarrow j$. Assigning a direction to each of the links splits the degree sequence $\mathbf{k}=\mathbf{k}_{\text {in }}+\mathbf{k}_{\text {out }}$, where $\mathbf{k}_{\text {in }}=$ $\left(k_{1}^{\text {in }}, k_{2}^{\text {in }}, \ldots, k_{N}^{\text {in }}\right)$ is the in-degree sequence and $k_{i}^{\text {in }}$ counts the number of links which are incident to node $i$, and respectively $\mathbf{k}_{\text {out }}=\left(k_{1}^{\text {out }}, k_{2}^{\text {out }}, \ldots, k_{N}^{\text {out }}\right)$, where $k_{i}^{\text {out }}$ counts the number of links that emanate from node $i$. Importantly, by construction one then has that the in-degree sequence of the HVG extracted from a given sequence is equal to the out-degree sequence of the HVG extracted from the time-reversed sequence, and therefore in order to assess time reversibility in $\mathscr{S}$, one can simply explore the statistical differences between the in-degree sequence and the out-degree sequence in the HVG (see $[29,30]$ for details and Fig. 3 for an illustration).

In [29] some of us proposed that the right-hand side Eq. (1) could actually be approximated by comparing the in and out degree distributions of $\operatorname{HVG}(\mathscr{S})$, as these are the $(m=1)$-point marginal distribution of the in and out degree sequences. Note that only needing to look at $m=1$ statistics is a substantial difference with respect to the benchmark method based on comparing $m$-gram statistics of the time series, as in this latter case by construction the statistics of 1-grams are invariant under time reversal, and irreversibility can only be checked for $m=2$ or higher. This resulting reduction will enhance our ability to effectively use the method in short sequences, as we will show below.

Whereas originally the HVG method only looked at strings of size $m=1$ (1-grams) in the degree sequence, one could of course further generalize this measure if it were needed to account for blocks of arbitrary size in the degree sequence, following the spirit of Eq. (1). For a block size $m$, let us consider strings of size $m$ within the in and out degree sequences such that with a little abuse of notation, $\mathbf{k}_{\mathrm{in}}^{m}=\left(k_{1}^{\mathrm{in}}, k_{2}^{\text {in }}, \ldots, k_{m}^{\text {in }}\right)$, and similarly for $\mathbf{k}_{\text {out }}^{m}$. Additionally, let $P_{m}^{\text {in }}(\mathbf{k})$ be the marginal distribution of the in-degree sequence blocks $\mathbf{k}_{\text {in }}^{m}$ [respectively for $P_{m}^{\text {out }}(\mathbf{k})$ ]. Then, we now define the HVG irreversibility of order $m$ as the Kullback-Leibler divergence between the in and out degree sequence blocks marginals

$$
\mathrm{KLD}_{m}(\text { in } \| \text { out })=\sum_{\mathbf{k}} P_{m}^{\text {in }}(\mathbf{k}) \ln \frac{P_{m}^{\text {in }}(\mathbf{k})}{P_{m}^{\text {out }}(\mathbf{k})} .
$$

This quantity is null if and only if the size- $m$ blocks are equally distributed in the (infinitely long) degree sequence, and are positive otherwise.

To summarize, the procedure to evaluate $\mathrm{HVG}$ reversibility in a time series $\mathscr{S}$ is as follows.

(i) From $\mathscr{S}$ we construct the directed HVG, and subsequently extract the in and out degree sequences $\mathbf{k}_{\text {in }}$ and $\mathbf{k}_{\text {out }}$. Note that for $\mathscr{S}^{*}, \mathbf{k}_{\text {in }}$ and $\mathbf{k}_{\text {out }}$ are interchanged. This procedure is applicable when $\mathscr{S}$ comes from both stationary and nonstationary processes alike.

(ii) To quantify for HVG reversibility (and HVG-entropy production), we make use of Eq. (2). Note that $m=1$ is the smallest nontrivial case here, as 1-grams of the degree sequence already incorporate temporal directionality (when using $m$-grams of $\mathscr{S}$ vs $\mathscr{S}^{*}, m=2$ is the simplest nontrivial case instead). 

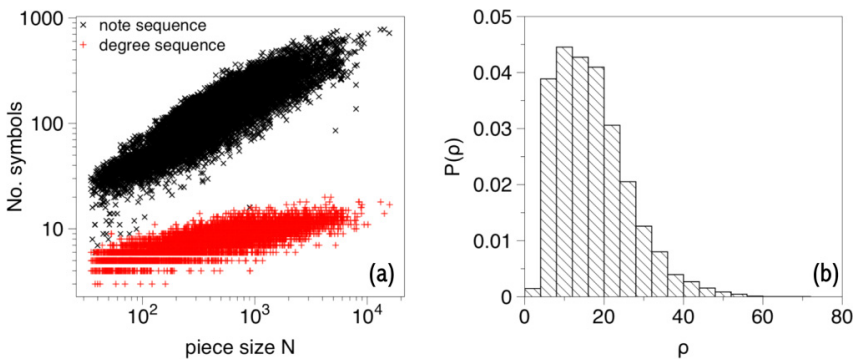

FIG. 4. [Panel (a)] Log-log plot of the minimum number of effective symbols needed to determine time irreversibility of a given musical composition, as a function of the size $N$ of the composition, using our HVG-based method (red plus) and using a standard 2-gram comparison in the note sequence (black crosses). We can see that the number of symbols in the method based on the note sequence is systematically in the same order of magnitude of total size of the sequence; hence finite size effects are too strong and make the method unapplicable. The method based on the HVG requires a substantially smaller number of symbols, orders of magnitude smaller than the sequence size, hence making this method useful in this data set. [Panel (b)] Histogram of the reduction factor $\rho$ (see the text) which compares the effective number of symbols needed in the HVG irreversibility method with respect to a standard method based on $n$-gram statistics. When we average over all the musical pieces, the average number of symbols is reduced 17 times, and this reduction can reach up to 75 times in some cases. This strongly reduces the finite size effects and hence makes the HVG method useful to explore time irreversibility in short sequences.

\section{On the number of symbols}

Once we have outlined the procedure to estimate the HVG irreversibility, we now consider the problem of working with short experimental sequences. First, it is important to note that the in and out degrees typically take values from a small alphabet-systematically smaller than the original musical note alphabet as we will show below-whose size increases at most logarithmically with $N$. This is because the probability that an arbitrary node in a HVG has a certain in and out degree $k$ typically decays exponentially fast with $k$ [41]. Moreover, it is important to recall that temporal irreversibility can already be assessed for $m=1$, that is, by only looking at the marginal distribution of the in and out degree sequences. Conversely, if we were to estimate time irreversibility directly on the note sequences, then we would at least need to consider strings of size $m=2$ consecutive notes. The effective number of symbols needed is therefore much larger than in our HVG setting. In the left panel of Fig. 4 we plot the effective number of symbols required to assess time irreversibility using HVG (red plus sign) and using a simple 2-gram comparison of the forward and backward note sequence (black crosses). We see that, in most of the cases, the number of note 2-grams is of the same order of magnitude as the size of the note sequence, hence making the standard approach useless. On the other hand, the approach based on HVG keeps the number of symbols needed to a bare minimum and is therefore useful in this context.

To give an additional quantitative idea of the symbol reduction given by the HVG method, let us define a reduction factor $\rho$ associated to a given musical piece as

$$
\rho=\frac{\text { total number of strings of size } m=2 \text { empirically found in the MIDI note sequence }}{\text { total number of different (in or out) degrees empirically found in the in and out degree sequence }} .
$$

In Fig. 4 we plot the histogram $P(\rho)$ estimated over our whole data set. The average reduction is about 17 , which means that our irreversibility method yields on average a $1700 \%$ reduction over the standard method based on counting the statistics directly on the note sequence. This implies that the finite size effects (subsampling)—which will appear due to the fact that the musical compositions are not exponentially larger than the number of symbols-will be contained in the case of the HVG method, thus enabling its use in applications where time series are short, such as in musical compositions.

Having said that, while small, finite size effects are still expected to emerge in finite time series, and because of that, the Kullback-Leibler divergence will always be positive (vanishing for reversible processes only asymptotically), which poses some interpretability problems. In what follows we introduce a confidence index whose aim is to solve this interpretability issue.

\section{Irreversibility ratio $\mathrm{IR}_{m}$ : A confidence index}

Let us define the $m$-order irreversibility ratio $\mathrm{IR}_{m}$ by standardizing the net irreversibility measure $\mathrm{KLD}_{m}$ (in||out) with respect to a null model where the original sequences are shuffled:

$$
\operatorname{IR}_{m}=\frac{\operatorname{KLD}_{m}(\text { in }|| \text { out })-\left\langle\mathrm{KLD}_{m}(\text { in }|| \text { out })\right\rangle_{\text {null model }}}{\sigma\left[\mathrm{KLD}_{m}(\text { in }|| \text { out })\right]_{\text {null model }}},
$$

where $\sigma$ is the standard deviation.

The process of standardizing is applied in order to be able to compare results across samples (musical pieces) with different sizes and marginal distributions, something which is recurrent in the musical compositions we analyze. More concretely, in the ideal situation of estimating irreversibility for infinitely long sequences, $\mathrm{KLD}_{m}$ (in||out) vanishes if and only if $P_{m}^{\text {in }}(\mathbf{k})=P_{m}^{\text {out }}(\mathbf{k})$, and therefore values different from zero would indicate that the sequence is statistically $\mathrm{HVG}$ irreversible. However, in practice this is not so clear-cut: the quantity $\mathrm{KLD}_{m}$ (in||out) for a reversible process is only asymptotically null (vanishing as the sample size goes to infinity), and will be finite (yet small) for finite samples due to statistical deviations in the estimation of joint probability functions $P\left(\mathbf{k}_{\text {in }}\right)$ and $P\left(\mathbf{k}_{\text {out }}\right)$. By standardizing this quantity with respect to a suitable null model, one can quantify the effective deviation of a given finite sample from the expected value if that finite sample was generated by a truly reversible process. The null model is built by taking 200 randomizations 


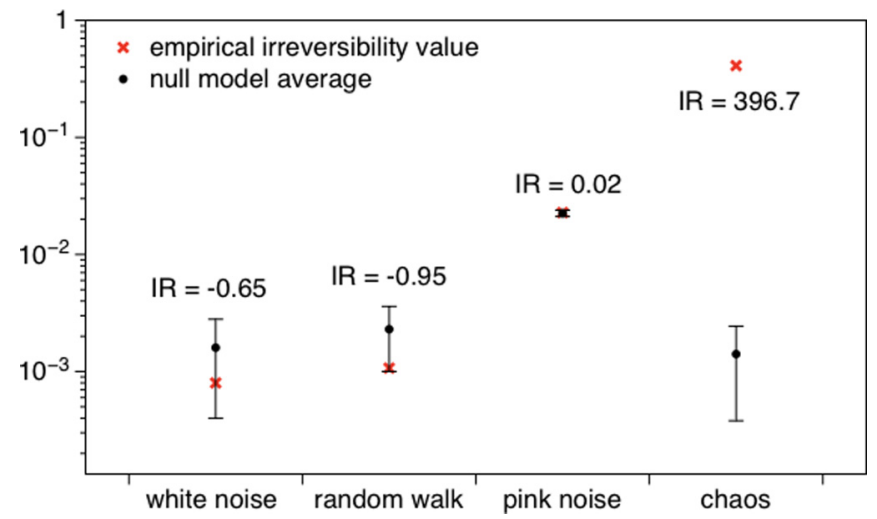

FIG. 5. Irreversibility ratios $\mathrm{IR}_{1}$ for three theoretically HVGreversible processes (uniform white noise, unbiased random walk, and pink noise) and one theoretically HVG-irreversible process [fully chaotic logistic map $x_{n+1}=4 x_{n}\left(1-x_{n}\right)$ ]. In every case we generate a time series of $N=10^{4}$ data points and symbolize with a vocabulary of $|\mathscr{V}|=100$ symbols. Red crosses correspond to the empirical irreversibility value of order 1 of the time series $\mathrm{KLD}_{1}$ (in||out), black dots correspond to the ensemble average irreversibility value $\left\langle\mathrm{KLD}_{1}(\mathrm{in}|| \text { out })\right\rangle_{\text {null model }}$ of $10^{3}$ null models constructed by randomizing the empirical time series (see the text), and the brackets correspond to \pm one standard deviation (note that the $y$ axis is in logarithmic scales). The irreversibility ratio of order 1 (see the text) decides whether the empirical time series is reversible $\left(\mathrm{IR}_{1} \leqslant 1\right)$ or irreversible $\left(\mathrm{IR}_{1}>1\right)$, and correctly predicts the true nature of the process. Interestingly, the empirical irreversibility value $\mathrm{KLD}_{1}$ (in||out) of pink noise is notably larger than for white noise or random walk, which could mislead to the suggestion that pink noise is irreversible. The irreversibility ratio asserts that this is not the case, suggesting that the difference in the raw values is due to differences in the specific marginal distribution of the underlying processes, which only yield spurious effects on the determination of temporal irreversibility.

of the sample sequence, and computing $\mathrm{KLD}_{m}$ (in||out) on each randomized sample. Then Eq. (3) measures the effective distance of a sample to its null model, in standard deviation (also known as "sigma") units. For instance, if $\mathrm{IR}_{m} \leqslant 1$ this means that the irreversibility value of a sample finite sequence is not statistically distinguishable from a finite sample of the same size and marginal distribution extracted from a truly reversible process. That does not necessarily mean that the process is $\mathrm{HVG}$ reversible, it only means that there is no statistical significance to assert otherwise. Similarly, if, e.g., $\mathrm{IR}_{m}=2$, this means that the HVG-irreversibility value of the sample is two standard deviations larger than the one expected for its (reversible) null model.

For illustration and validation, in Fig. 5 we plot the raw HVG-irreversibility value $\mathrm{KLD}_{1}$ (in||out) (red crosses) and $\mathrm{IR}_{1}$ for time series extracted from four synthetic dynamical processes: (i) white noise, (ii) random walk, (iii) pink noise, and (iv) a fully chaotic process.

Process (i) is an (uncorrelated) uniform white noise. This is a stationary stochastic process with deltalike autocorrelation function; it is statistically reversible and $\mathrm{HVG}$ reversible. The method correctly identifies this character as $\mathrm{IR}_{1}<1$ in this case.
Process (ii) is an unbiased discrete random walk $x(t+$ $1)=x(t)+\eta$, where $\eta \sim$ uniform $(-1 / 2,1 / 2)$. Interestingly, while this is a nonstationary process and thus could be seen as irreversible, it can be shown to be so-called HVG stationary [30] and explored adequately within the HVG framework, as HVG reversible with $\mathrm{IR}_{1}<1$. This is indeed convenient as Brownian particles do not produce entropy on average, so in the context of $\mathrm{HVG}$ reversibility, we recover the relation between reversibility and entropy production in this nonstationary process.

Process (iii) is a linearly correlated noise with a $1 / f$ spectrum. This is again, by definition, a time reversible process, and is a paradigmatic (stationary) stochastic process to describe music from the pioneering works of Voss and Clarke $[1,2]$. Also in this case we correctly detect the reversible character as $\mathrm{IR}_{1}<1$.

Finally, process (iv) is a deterministic chaotic process generated by a fully chaotic logistic map $x_{t+1}=4 x_{t}\left(1-x_{t}\right)$. This process is dissipative and statistically time irreversible (and HVG irreversible), as certified by $\mathrm{IR}_{1}>1$.

Another important aspect is to understand how $\mathrm{IR}_{m}$ is affected by the time series length $N$. Intuitively, if the underlying process is $\mathrm{HVG}$ reversible, then $\mathrm{KLD}_{1}$ (in||out) should be similar to its null model (within its uncertainty) and both measures should be decaying with the same trend as $N$ increases, and therefore one should expect $\operatorname{IR}_{m}<1$, independent of $N$. If, on the other hand, the underlying process is HVG irreversible, then $\mathrm{KLD}_{1}$ (in||out) should be systematically larger than zero and remain positive as we increase $N$. Since the null model of this process is $\mathrm{HVG}$ reversible, its irreversibility value should decrease as $N$ is increased; we thus expect $\mathrm{IR}_{m}$ effectively to increase with $N$ without bounds. This only means that for an HVG-irreversible process, whereas for short time series the distinguishability is small ( $\mathrm{IR}_{m}$ close to 1 ), as we increase the series size it is systematically easier to ascertain that the series (and process) is $\mathrm{HVG}$ irreversible. In some sense, $\mathrm{IR}_{m}$ is therefore a measure of confidence.

We illustrate this dependence on series size in Fig. 6, where in panels (a) and (b) we consider a truly reversible process [white noise $\left.x_{t}=\eta, \eta \sim \operatorname{Uniform}(0,1)\right]$ and in panels (c) and (d) we consider a truly irreversible process [a fully chaotic logistic map $\left.x_{t+1}=4 x_{t}\left(1-x_{t}\right)\right]$. Results indeed confirm our discussion above.

Once we have illustrated how the irreversibility ratio works, let us introduce a classification of net HVG irreversibility for a given musical piece as follows.

Definition III..2. A musical piece is defined as HVG reversible (or simply reversible) if $\mathrm{IR}_{1} \leqslant 1$. If $1<\mathrm{IR}_{1} \leqslant 4$ we say that the process is $\mathrm{HVG}$ irreversible with weak confidence. If $4<\mathrm{IR}_{1} \leqslant 10$, we say that it is HVG irreversible with strong confidence, and if $\mathrm{IR}_{1}>10$ we say that the musical piece is HVG irreversible with extreme confidence.

A similar classification can be defined for higher orders $m>1$; however, this is not needed for this work since $\mathrm{IR}_{1}$ shows strong correlation with $\mathrm{IR}_{m>1}$ for most of the musical pieces considered, hence it will be enough to concentrate our analysis on $m=1$. Incidentally, note that since blocks are extracted from the HVG's degree sequence (not directly from 

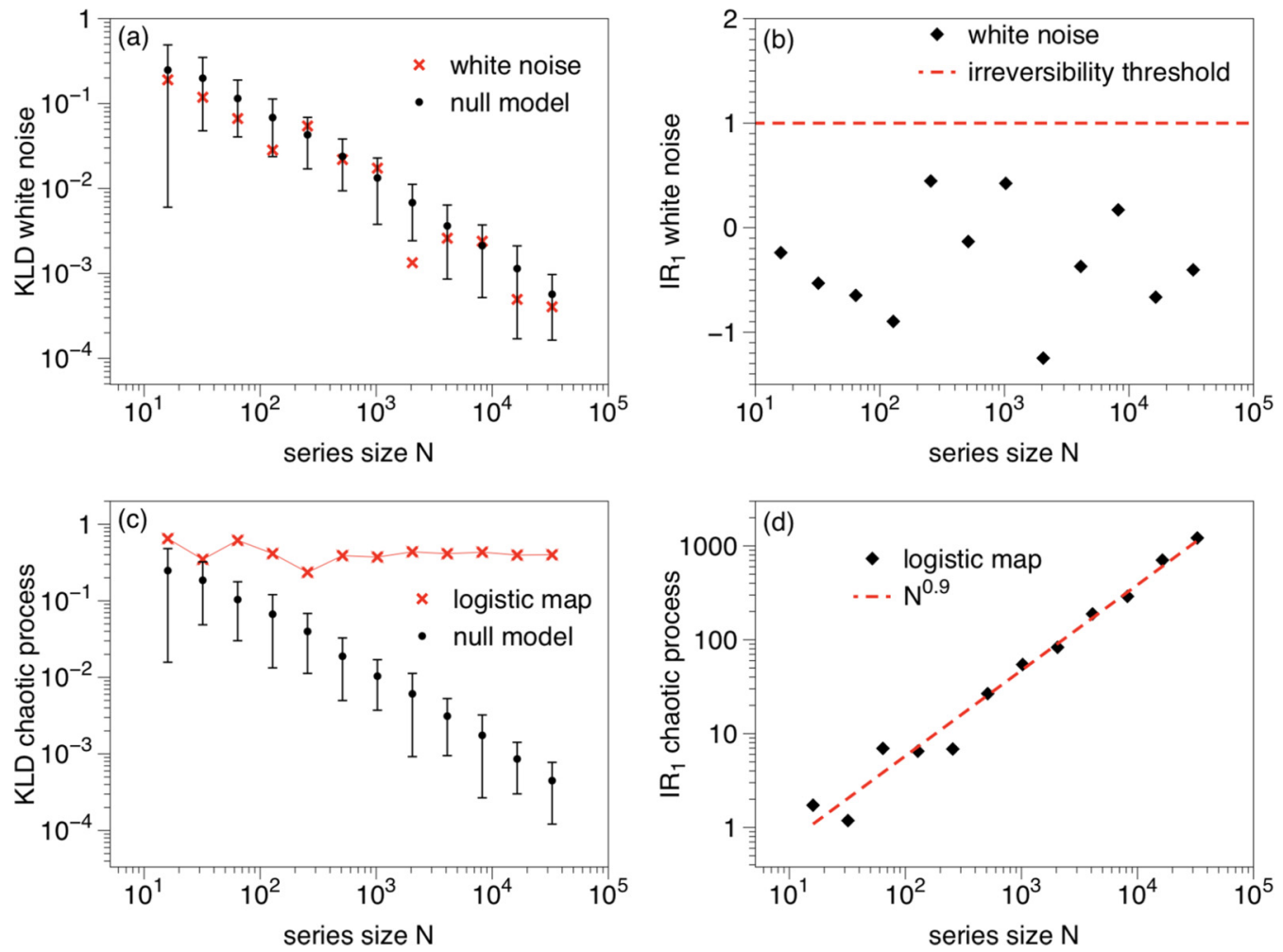

FIG. 6. (a) Raw order-1 irreversibility value $\mathrm{KLD}_{1}$ (in||out) of a time series of size $N$ generated by a reversible white noise process $x_{t}=$ $\eta, \eta \sim$ Uniform $(0,1)$ (red crosses). For comparison, the results of a null model (mean \pm one standard deviation) where we randomize the original time series 200 times and compute the irreversibility value is shown in black solid circles. The raw irreversibility value decreases without bounds in a fashion similar to the null model, certifying that positive irreversibility values are here only due to finite size effects which vanish as $N$ increases. (b) Order-1 irreversibility ratio $\mathrm{IR}_{1}$ for the process depicted in panel (a), certifying that the time series is not distinguishable from a reversible process for any time series size. (c) Similar to (a), for a time series of size $N$ generated by an irreversible, fully chaotic logistic map $x_{t+1}=4 x_{t}\left(1-x_{t}\right)$ (red crosses). The raw irreversibility value is always positive and stabilizes for $N>10^{3}$. The null model is by definition reversible, and its irreversibility value is only positive due to finite size effects, hence decreasing as $N$ increases. Distinguishability therefore increases with $N$. (d) Similar to (b), for the chaotic case, certifying that the process is irreversible and that the level of confidence increases without bounds.

the sequence of notes), in principle the HVG-irreversibility measure at $m=1$ already gathers temporal information of different time scales.

Summing up, we have shown that the concept of HVG reversibility is better suited than the standard concept of statistical time reversibility to investigate the arrow of time in stationary and nonstationary processes [30], and HVG is a useful approach when time series are short. We can safely conclude that $\mathrm{IR}_{m}$ is a measure that quantifies our certainty that the time series under study was generated by an HVGirreversible process. To compute such confidence, the size of the series (and the resulting finite-size effects) and the specific shape of the marginal distribution of the signal must be taken into account in order to provide a quantifier which is not affected by these variables. On the other hand, and once the analysis based on $\mathrm{IR}_{m}$ leads us to conclude that the process is indeed HVG irreversible, we will then use $\mathrm{KLD}_{m}$ (in||out) as a bound for the true (thermodynamic) entropy production rate of the process.

In the next sections we will compute HVG-reversibility metrics, but in order to lower down verbosity we will refer to it indistinctively as either HVG reversibility or just reversibility.

\section{RESULTS}

\section{A. Irreversibility}

To start, we have explored the confidence of time series irreversibility - as quantified by $\mathrm{IR}_{m}$ - for all the pieces considered in this work. In the left panel of Fig. 7 we depict in semilog scales the normalized histograms $P\left(\operatorname{IR}_{m}\right)$ for $m=$ $1,2,3$. The orange area denotes the region $\operatorname{IR}_{m}>1$, showing that a large percentage of pieces are irreversible (HVG irreversible) at all $m$ orders, with varying degree of confidence.

A second observation is that the histograms for $m=2$ and $m=3$ are indeed very similar. In order to further understand to what extent the three measures $\mathrm{IR}_{1}, \mathrm{IR}_{2}$, and $\mathrm{IR}_{3}$ are correlated, we have computed the Pearson correlation coefficient between $\mathrm{IR}_{m}$ and $\mathrm{IR}_{m-1}$ (see Appendix A, Fig. 14), showing that indeed we find a strong correlation between all of them. The interpretation of this finding is twofold: we can first argue that, in the context of classical music and the database analyzed in this work, higher-order irreversibility is irrelevant, and all the structure can be efficiently captured by order- 1 HVG reversibility. Second, this also means that from now on we can safely focus our analysis on $m=1$, which is notably faster to estimate. 
(a)

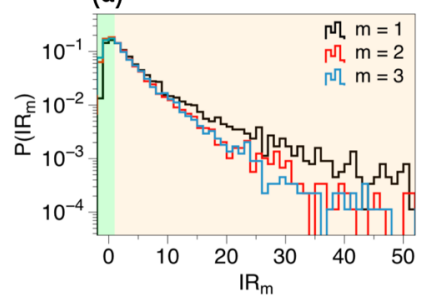

(b)

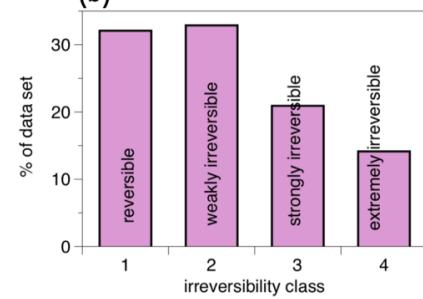

(c)

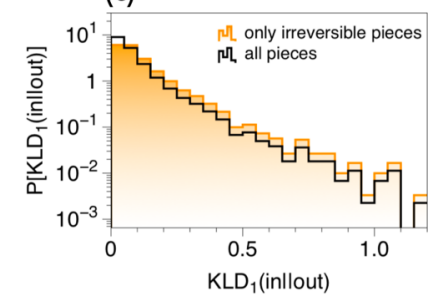

FIG. 7. [Panel (a)] Estimated normalized histogram of irreversibility ratios $\operatorname{IR}_{m}(m=1,2,3)$ for all pieces in our data set, in a semilog plot. The green area highlights the region where pieces are reversible. A large portion of the pieces are indeed generated by an irreversible process. [Panel (b)] Abundance per irreversibility confidence class. [Panel (c)] Estimated normalized histogram of raw irreversibility values $\mathrm{KLD}_{1}$ (in||out), for all pieces (black) and only those pieces which have previously been certified as irreversible (i.e., IR $\left.1>1\right)($ orange). A linear binning of size 0.05 has been performed.

The abundance of each irreversibility confidence class is depicted in the middle panel of Fig. 7, showing that all four classes have a notable representation, where only about $30 \%$ of the whole data set complies with a reversible structure. In the right panel of the same figure we have plotted in semilog the normalized histogram of $\mathrm{KLD}_{1}(\mathrm{in}|| \mathrm{out})$, for all pieces (black curve) and only for those pieces which have previously been certified to be irreversible $\left(\mathrm{IR}_{1}>1\right)$. In both cases, values mostly concentrate in the interval $[0,1]$, and most of the pieces with high irreversibility value [e.g., $\mathrm{KLD}_{1}(\mathrm{in}||$ out $)>$ $0.1]$ correspond to those pieces previously checked to be indeed irreversible according to the irreversibility ratio criterion. Distributions decay rapidly in both cases, highlighting that this measure is highly concentrated towards the left end of the spectrum.

We can now concentrate on the subset of pieces which are certified to be irreversible $\left(\mathrm{IR}_{1}>1\right)$, and we can rank both composers and pieces according to their net irreversibility value. For composers, this ranking is shown in Table I. Since irreversibility is linked with entropy production, we could provocatively say that this is a ranking of the composers which, on average, have a "more out of equilibrium" compositional process, i.e., the composers whose compositional style dissipates more energy and on average produces more entropy accordingly. A similar ranking for the most irreversible pieces is depicted in Table II.

In order to explore the evolution of irreversibility over different periods, in Fig. 8 we plot the values of $\mathrm{IR}_{1}$ (top) and $\mathrm{KLD}_{1}$ (in||out) (bottom) for all pieces as a function of the date of birth of the composer of the piece. Interestingly, the irreversibility ratios seem to fluctuate in a nonrandom way. To highlight such modulation, in panel (b) of Fig. 8 we plot the same data and we add as orange dots the result of a linear binning (bins of $45 \mathrm{yr}$ size). The green dashed dot corresponds

TABLE I. Ranking of most irreversible composers.

\begin{tabular}{lcccc}
\hline \hline Rank & Composer & $\left\langle\mathrm{KLD}_{1}(\right.$ in $\mid$ out $\left.)\right\rangle$ & $\mathrm{DOB}$ & Number of pieces \\
\hline 1 & Bizet & 0.28 & 1838 & 21 \\
2 & Mahler & 0.19 & 1860 & 28 \\
3 & Dowland & 0.18 & 1563 & 61 \\
4 & Desprez & 0.178 & 1521 & 34 \\
5 & Paganini & 0.155 & 1782 & 24 \\
\hline \hline
\end{tabular}

to the best fit of a cubic polynomial to the binned data, highlighting an apparent modulation inside musical compositions over different time periods. In panel (c) of the same figure, instead of displaying the $\mathrm{IR}_{1}$ for each piece, we average per composer and plot the average irreversibility ratio $\left\langle\mathrm{IR}_{1}\right\rangle$ for each composer, as a function of the composer's date of birth. A cubic polynomial is once more fitted to this data, again highlighted the modulated trend. Superposed to this figure we have highlighted the different musical periods (Renaissance, Baroque, Classic, Romantic, and early Modern). Since $\mathrm{IR}_{1}$ has a strong dependence on piece size and this is not the case for $\mathrm{KLD}_{1}$ (in||out) (see Appendix C), a similar analysis is then replicated in panels (d)-(f) of Fig. 8 for the irreversibility value $\mathrm{KLD}_{1}$ (in||out), tracking how irreversibility values (i.e., entropy production) evolve over time. The modulated trend along the different musical periods is not present anymore. We can only conclude that over different periods, different composers have systematically produced pieces which were irreversible, but the effect of different styles in the particular value of irreversibility is less clear.

\section{B. Nonlinearity index $\xi$}

To complement the irreversibility analysis of musical compositions, in a second step we consider the temporal arrangement of note sequences within each piece. While it has been extensively certified that music evidences long-range temporal correlations with (typically) a heavy tailed power spectrum $[1,3,7,8]$, less is known about nonlinear correlations. However, recent studies have reported evidence of nonlinear correlations in musical pieces and discussed their possible relevance in their structure [20].

In order to assess the amount of nonlinear temporal correlations, we define a nonlinearity index $\xi$ inspired in the index previously introduced in [42] by computing the significance (and the amount of nonlinearity) in the magnitude detrended fluctuation analysis (MDFA) of each musical piece and its Fourier-fixed surrogates (null model with linear correlations). Basically for the calculation of $\xi$ we compare both MDFA computations (original and surrogates) in terms of the local slopes of a fitting polynomial $(\hat{y})$ for the function $F(s) / s$ (see Appendix B for details):

$$
\xi=\frac{1}{N_{w s}} \sum_{i=1}^{N_{w s}} \frac{\left|\hat{y}^{\prime}\left(x_{i}\right)-\left\langle\hat{y}_{s}^{\prime}\left(x_{i}\right)\right\rangle_{\mathrm{sur}}\right|}{\sigma\left(\hat{y}_{s}^{\prime}\left(x_{i}\right)\right)_{\mathrm{sur}}},
$$


TABLE II. Ranking of most irreversible pieces.

\begin{tabular}{|c|c|c|c|c|c|}
\hline Rank & Piece & $\mathrm{KLD}_{1}($ in $\mid$ out $)$ & Composer & DOB & Piece length \\
\hline 1 & Silhouettes 09 & 5.33 & Dvorak & 1841 & 269 \\
\hline 2 & Gadfly $97 \mathrm{a}, 3$ & 3.82 & Shostakovitch & 1906 & 492 \\
\hline 3 & Swan Lake (Act4,25 Entract e) & 3.57 & Tchaikovsky & 1840 & 179 \\
\hline 4 & Kinderszenen 1510 & 2.45 & Schumann & 1810 & 199 \\
\hline 5 & Messiah 14 & 2.22 & Handel & 1685 & 96 \\
\hline
\end{tabular}

where $N_{w s}$ is the total number of windows of size $s, \hat{y}^{\prime}\left(x_{i}\right)$ is the first derivative of the polynomial evaluated at the $i$ th window of size $\left[x_{i}=\log \left(s_{i}\right)\right]$, and $\left\langle\hat{y}_{s}^{\prime}\left(x_{i}\right)\right\rangle_{\text {sur }}$ and $\sigma\left(\hat{y}_{s}^{\prime}\left(x_{i}\right)\right)_{\text {sur }}$ represent the mean and variance of the slopes in the ensemble of surrogates at the $i$ th window size, respectively (see Appendix B for more details). Surrogates were generated with the iterative amplitude adjusted Fourier transform (IAAFT) algorithm $[43,44]$, preserving the marginal distribution and the power spectrum of the original piece.

By construction, $\xi \leqslant 1$ would indicate that the signal only evidences (at most) linear correlations, whereas if $\xi>1$ then the signal has correlations of nonlinear nature (not reflected in the power spectrum), and the larger $\xi$ the stronger they are [44]. In Fig. 9 we measure the nonlinearity index $\xi$ for all the pieces considered in the database. The left panel displays its frequency histogram, certifying that indeed a large majority of pieces display a high nonlinearity index. In the right panel of the same figure we plot $\xi$ as a function of the piece composer's date of birth. Notably, we find that a substantial amount of all musical compositions considered display different degrees of nonlinearity, and the similarity of this panel with panels (a) and (d) in Fig. 8 is suggestive. Note that though evidence for different profiles of nonlinear correlations in music scores has been reported previously [20], a specific index such as $\xi$, able to quantify the amount of nonlinearity in a signal, was lacking.

\section{Irreversibility vs nonlinearity}

In order to understand and link the concept of time irreversibility to nonlinearity, we have investigated to what extent the irreversible character holds when the pieces keep their linear correlations structure but are randomized otherwise. For each piece we have therefore constructed a surrogate piece where the linear correlation structure (power spectrum) is maintained by applying the same technique to the one described previously (IAAFT surrogates), and we then compared $\mathrm{IR}_{1}$ in both cases. In the left panel of Fig. 10 we compare the histograms of $\mathrm{IR}_{1}$ for all pieces and for all surrogate pieces. Interestingly, in the case of the surrogates a large
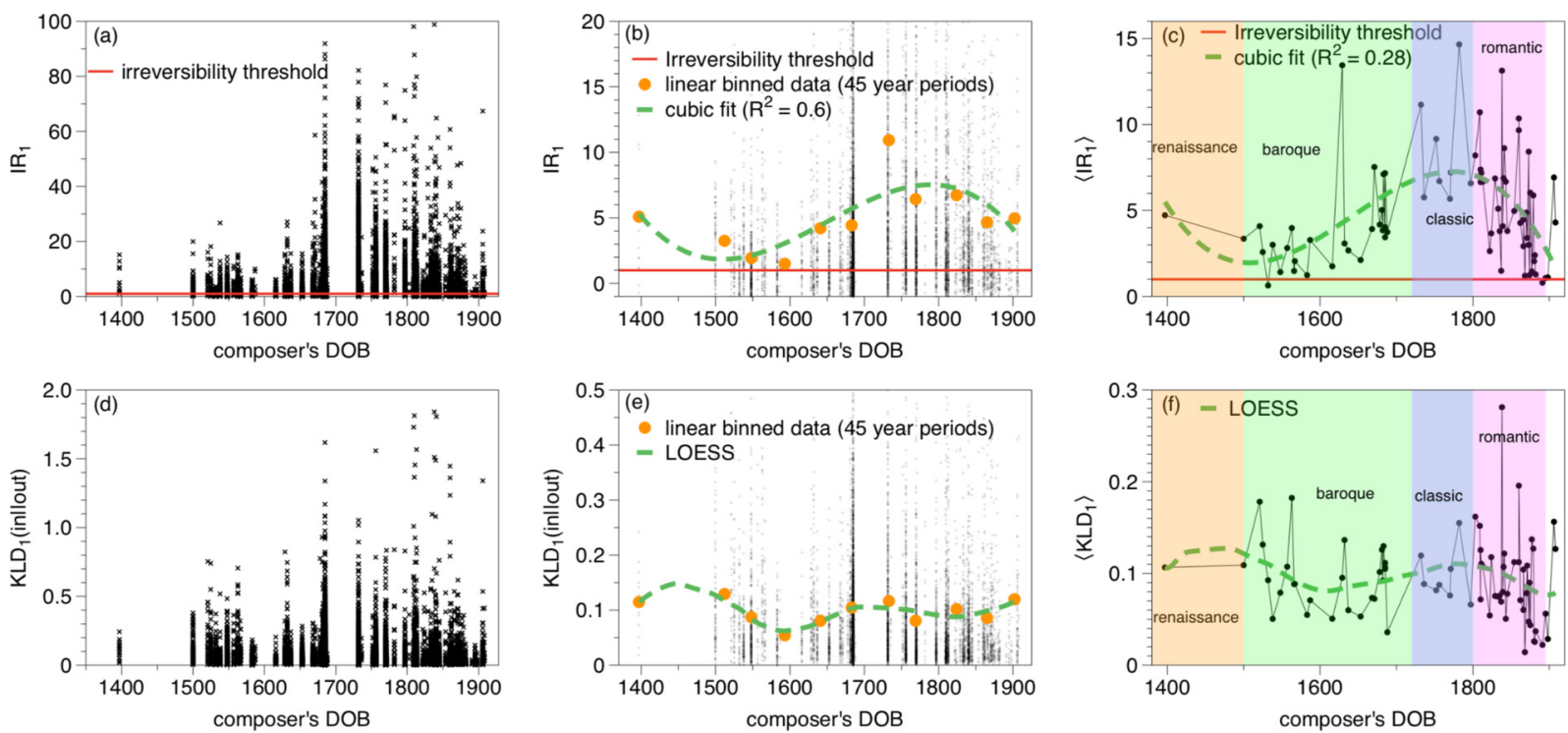

FIG. 8. Evolution of irreversibility over different musical periods. ( a) Irreversibility ratio $\mathrm{IR}_{1}$ vs the composer's date of birth of each piece (crosses), for all pieces considered. The red solid line denotes the irreversibility threshold, suggesting that most of the pieces are statistically irreversible. (b) Similar to panel (a), but where a cubic function (green dashed line) has been fitted to data after a linear binning (in periods of $45 \mathrm{yr}$ ) has been performed. The fit highlights a modulating trend by which compositions net irreversibility confidence tend to vary over different periods. (c) Similar to the (a) panel, but where irreversibility ratio is averaged over all the pieces of the same composer (one data point per composer). The modulating trend is still present, and for reference we also include the different musical periods (Renaissance, Baroque, Classical, Romantic, and early Modern). (d) Similar to (a), but where we plot the raw irreversibility value $\mathrm{KLD}_{1}$ (in||out) as a proxy for the entropy production rate per piece. Qualitatively the same pattern as in (a) emerges. (e) Similar to (b) but where we plot KLD $($ in||out). Orange dots correspond to a linear binning and can be interpreted as the average entropy production rate of all pieces over each 45 -yr period. This rate is maintained approximately constant over time. The fit is a local estimated scatterplot smoothing (LOESS). (f) Similar to (c), but averaging the value $\mathrm{KLD}_{1}$ (in||out) for all pieces of each artist separately. This gives us a proxy for an average entropy production rate per composer. 

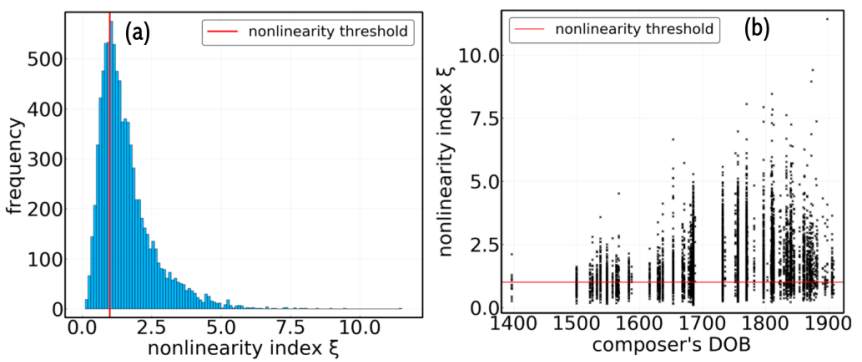

FIG. 9. Nonlinearity index. [Panel (a)] Histogram of the nonlinearity index $\xi$ (see the text) for all pieces considered. [Panel (b)] Nonlinearity index $\xi$ as a function of the composer's date of birth.

percentage of the pieces now display HVG reversibility (see the middle and right panel of the same figure for a graphical demonstration of the irreversibility loss induced by surrogating the signals). This result can be explained as follows: Gaussian linear processes with a prescribed power spectrum are indeed reversible. We can understand surrogate pieces as stochastic processes with a prescribed power spectrum (the same as the original piece) but no additional temporal correlation kernels beyond the linear one, and therefore by construction surrogates should be time reversible. All in all, these results point to the fact that time series irreversibility has a connection to nonlinearity.

Such connection is reinforced by the similarity between the right panel of Fig. 9 (nonlinearity index $\xi$ ) and panels (a) and (d) of Fig. 8 where we display the values of $\mathrm{IR}_{1}$ and $\mathrm{KLD}_{1}$ (in||out) for all pieces as a function of the date of birth of the composer of the piece. Since the method we use to estimate the amount and significance of nonlinearity has been proved to depend on the length of the series [45] (see Appendix B), for the estimation of the statistical dependence between nonlinearity and any other property we use the mean value for the local slopes in the MDFA function, which is also related with the amount of nonlinearity and is less dependent on the length of the series [42] (see Appendix B):

$$
\left\langle\hat{y}^{\prime}(x)\right\rangle=\frac{1}{N_{w s}} \sum_{i=1}^{N_{w s}} \hat{y}^{\prime}\left(x_{i}\right),
$$

where $\hat{y}(x)$ is the polynomial fitted for $\log [F(s) / s]$ used for the calculation of the index $\xi$ [quantities are determined before the significance test in Eq. (4)]. In order to quantify the apparent correlation between nonlinearity and irreversibility, we have computed the mutual information between the amount of nonlinearity proxy $\left\langle\hat{y}^{\prime}(x)\right\rangle$ and $\mathrm{KLD}_{1}$ (in||out) for all pieces and also for the subset of irreversible pieces, using the discrete mutual information between two random variables $X, Y$ :

$$
\operatorname{MI}(X ; Y)=\sum_{y \in \mathscr{Y}} \sum_{x \in \mathscr{X}} p(x, y) \ln \left(\frac{p(x, y)}{p(x) p(y)}\right) .
$$

We define a mutual information confidence index, in the same fashion as nonlinearity and irreversibility indexes, by subtracting the average $\langle\mathrm{MI}\rangle_{\text {null }}$ and dividing by the standard deviation $\sigma(\mathrm{MI})_{\text {null }}$ of a null model:

$$
\mathrm{MI}_{\text {index }}=\frac{\mathrm{MI}-\langle\mathrm{MI}\rangle_{\text {null }}}{\sigma(\mathrm{MI})_{\text {null }}},
$$

where the null model is generated random shuffling the elements of one of the variables. The MI indexes are computed by sampling 1000 realizations of the null model. We chose this statistical dependence measure because it captures all kinds of correlations (linear and nonlinear) that would be relevant for this case, in contrast with other measures that assume linear dependencies (e.g., Pearson or Spearman coefficients).

Results of $\mathrm{MI}_{\text {index }}$ are plotted in Fig. 11. We conclude that HVG irreversibility is indeed intimately related in musical compositions with the presence of nonlinear correlations in the signal. Noting that HVG reversibility is a proxy for entropy production, this relation manifests a link between a physical concept (dissipation and entropy production) and a statistical one (nonlinear temporal correlations), hence giving a physical interpretation to the latter.

\section{Interval asymmetry}

With the aim of linking the patterns observed in terms of irreversibility and nonlinearity with a quantity of musical significance, we finally consider the statistics of intervals. An interval is defined as the distance between two consecutive notes in a musical piece. For a given sequence of $N$ notes $\left(n_{t}\right)_{t=1}^{N}$, one can define its respective sequence of intervals as $\left(i_{t}\right)_{t=1}^{N-1}$, with $i_{t}=n_{t+1}-n_{t}$, whose properties have been previously analyzed [46-50]. One particular known result is that small intervals are predominantly descending, while large
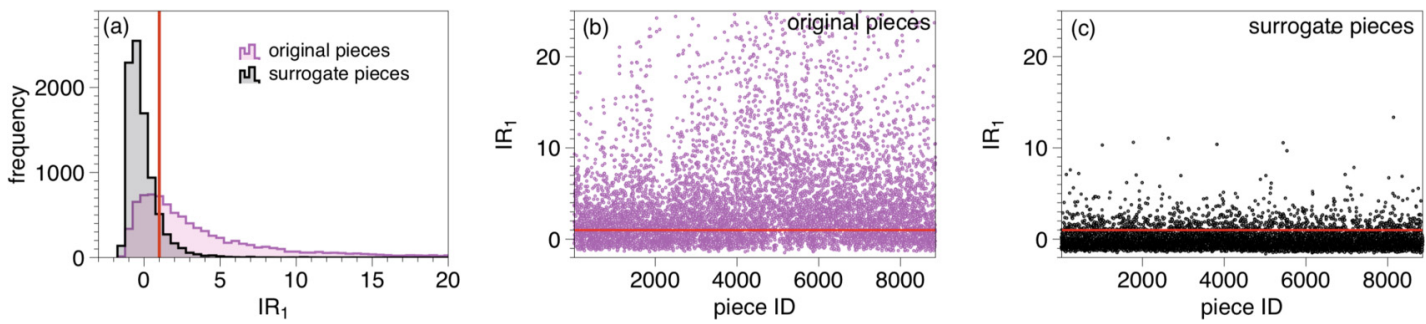

FIG. 10. Irreversibility is closely related to nonlinearity. (a) Histogram of $\mathrm{IR}_{1}$ for all pieces considered, compared to a similar histogram applied on surrogate pieces where the linear correlation structure (power spectrum) is maintained and other (nonlinear) temporal correlations are removed. We observe that a very large percentage of the surrogate pieces are time reversible, in agreement with the theory. (b),(c) $\mathrm{IR}_{1}$ of each of the 8856 pieces considered, where we clearly see that many of these are confidently irreversible. Their surrogates, where only linear temporal correlations are maintained, are however largely reversible: the construction of the surrogates removes in most of the cases the irreversible character of the original pieces, further demonstrating that statistical irreversibility is a property that cannot be explained by linear correlations (power spectrum) and ought to be related to nonlinear traits. 


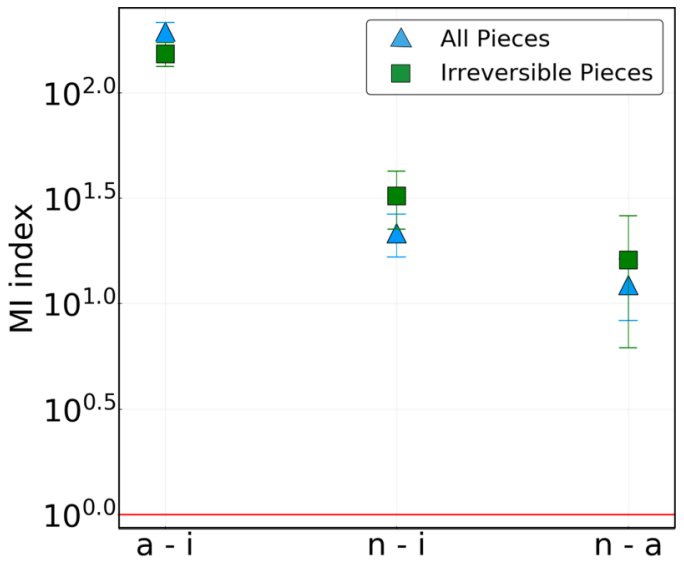

FIG. 11. Mutual information index $\mathrm{MI}_{\text {index }}$ between irreversibility, nonlinearity, and asymmetry. Significance for the mutual information between the three properties: irreversibility $\left[\mathrm{KLD}_{1}\right.$ (in || out)] as defined in Eq. (2), nonlinearity $\left[\left\langle\hat{y}^{\prime}(x)\right\rangle\right]$ defined in Eq. (5), and asymmetry $\left(D_{\uparrow \downarrow}\right)$ as defined in Eq. (8). The red solid line determines the threshold above which there is a statistically significant correlation between indices (the larger $\mathrm{MI}_{\text {index }}$, the stronger). On the $x$ axis: (a-i) asymmetry vs irreversibility, (n-i) nonlinearity vs irreversibility, and (n-a) nonlinearity vs asymmetry. Triangles represent the MI significance for all the pieces, while squares display the same quantities computed only considering the pieces which are confidently irreversible $\left(\mathrm{IR}_{1}>1\right)$. The error bars represent the $95 \%$ confidence intervals computed by bootstrapping with replacement.

ones are typically ascending [22]. This property introduces an asymmetry in the distribution of intervals which, intuitively, would contribute to the heterogeneity of the joint distributions of consecutive notes in melodic sequences. In order to investigate the relation between interval asymmetry, irreversibility, and nonlinearity, we first look at the interval distribution of the set of pieces we studied. We only consider intervals shorter than or equal to an octave (12 semitones), since larger intervals are less frequent. Figure 12 displays the interval distributions observed in the complete set of original pieces (left panel) and for the ensemble of surrogates generated previously in the nonlinearity test (right panel). The most evident difference between both distributions is the frequency of the zero interval (when the note keeps the same value), which is lower for the original pieces. The claim of the difference between ascending and descending intervals holds in our data; the intervals $-4,-3,-2,-1$ are more frequent than $4,3,2,1$, respectively, whereas the interval 5 is more frequent than -5 . However, it is not clear that for larger intervals the claim holds. Since the interval distributions are computed for the whole corpus (over 8000 pieces) and not for individual pieces we cannot relate directly this distribution asymmetry with irreversibility. To explore the interval statistics on individual pieces and the possible relation of the asymmetry with nonlinearity and irreversibility we measure the difference between positive (ascending) and negative (descending) intervals $\left(D_{\uparrow \downarrow}\right)$ for a given piece:

$$
D_{\uparrow \downarrow}=\frac{\left|I_{\uparrow}-I_{\downarrow}\right|}{I_{\uparrow}+I_{\downarrow}},
$$

where $I_{\downarrow}$ is the number of negative intervals (when $i_{t}<0$ ) and $I_{\uparrow}$ the number of positive ones $\left(i_{t}>0\right)$. If the interval distribution of a piece is symmetric (same number of ascending and descending intervals) then the difference $D_{\uparrow \downarrow}=0$ and if there is only one direction in the melody (ascending or descending) the difference would be $D_{\uparrow \downarrow}=1$.

Results for the interval difference $\left(D_{\uparrow \downarrow}\right)$ are shown in Fig. 13, where $D_{\uparrow \downarrow}$ is determined for all the original pieces and for their ensemble of surrogates. The first panel (left) shows the distributions for the values of interval difference $\left(D_{\uparrow \downarrow}\right)$; the second panel (right) is a plot of the average value of $D_{\uparrow \downarrow}$ for each composer. We systematically find a strong interval asymmetry in a large part of the musical pieces. According to the mutual information index (Fig. 11) $D_{\uparrow \downarrow}$ indeed strongly correlates with the HVG-irreversibility metric $\mathrm{KLD}_{1}$ (in||out), and to a lesser extent with the nonlinearity metric $\left\langle\hat{y}^{\prime}(x)\right\rangle$, thus concluding that the irreversibility and nonlinearity traits observed in musical compositions can indeed be narrowed down to musical concepts.
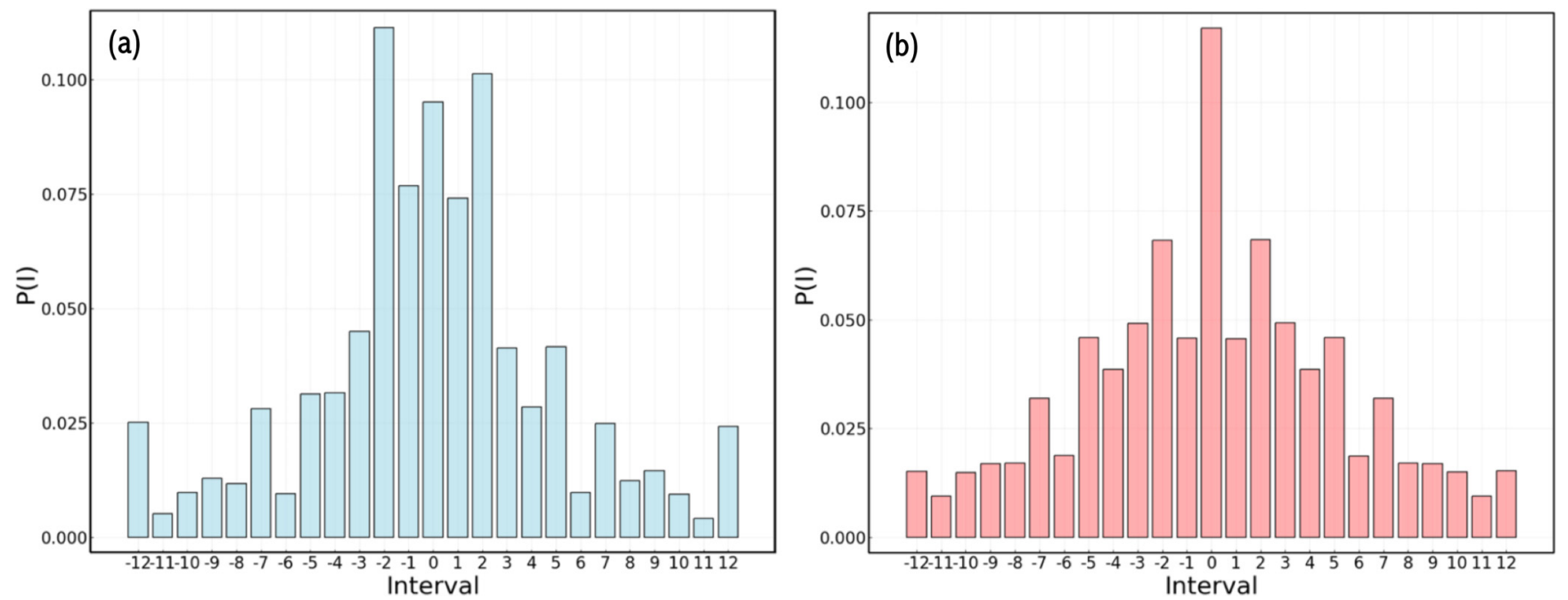

FIG. 12. Interval distributions. [Panel (a)] Distribution of the intervals for all the pieces in this study. [Panel (b)] Distribution of the intervals for the ensemble of surrogates constructed in the nonlinearity test. 

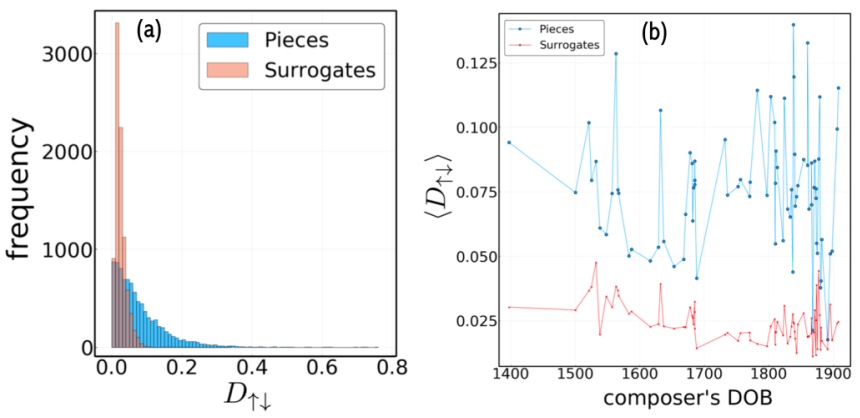

FIG. 13. Interval difference. [Panel (a)] Distributions of the values of $D_{\uparrow \downarrow}$ for all the pieces in the study. [Panel (b)] Mean value of the interval difference for each composer.

\section{DISCUSSION}

The concepts of irreversibility, directionality, and their musical implications have been qualitatively and allegorically evoked in music theory under multiple forms. For instance, temporal directionality has been established in terms of irreversible relations of "before" and "after" [21]. Indeed, according to Hastey [21], for some theorists "directionality arises in the compositions only through our ability to predict the future course of events. In tonal music a leading tone or passing dissonance implies an expected resolution. Such expectations whether realized or not, constitute in our imagination goals toward which the music is directed". On the other hand, irreversibility was one of the main concerns of musicians such as Anton Webern, one of the exponents of atonality and serialism. Similarly, allegoric relations to thermodynamics, entropy, and information theory have been previously explored by musicians, e.g., Iannis Xenakis, father of stochastic music [51].

The first aim of this work is to provide a quantitative study of the above concepts. For that, we used tools from statistical physics, nonlinear dynamics, and graph theory to characterize a large database of 8856 classical music compositions ranging over five centuries, encompassing pieces from 77 composers from the Renaissance up to the early Modern period. We showed that, indeed, time irreversibility can not only be measured, but is also pervasive, as we found that over two-thirds of these compositions indeed display this signature, leading us to conclude that this is a common trait of tonal music, in agreement with Hastey. The fact that a signal is time irreversible points to an emergent time arrow. Furthermore, we found evidence which suggests that at least some of the amount of such statistical property could be explained here in the light of another purely musical quantifier-the interval asymmetry-further supporting the ideas of Xenakis around thermodynamics and music. Indeed, since time irreversibility (as quantified here by the KullbackLeibler divergence between the in and out order- $m$ degree distributions of the signal's horizontal visibility graphs) is a proxy for the amount of thermodynamic entropy produced by a physical signal, exploring time irreversibility in musical compositions allows us to quantify the process of composition in out-of-thermodynamic-equilibrium terms.

The second aim of this work is to challenge the traditional vision whereby the complexity of music emanates from its linear temporal correlation structure, using $1 / f$ noise as a paradigm offering a balance between predictability and surprise and justifying its enjoyment. As a matter of fact, Gaussian stochastic processes with a linear correlation structure, such as $1 / f$ noise, are time reversible, and thus cannot explain the richer structure predominantly found in classic music, which is predominantly time irreversible. To shed further light into this result, we explored the amount of nonlinearity in the correlation structure, which by definition is also absent in $1 / f$ noise. We found that not only musical compositions display notable amounts of nonlinearity, but also that irreversibility is correlated with nonlinearity. This unravels the unforeseen notion that nonlinearity is related to a preferential time arrow, and unveils that our three quantifiers (time irreversibility as described by the irreversibility index $\mathrm{IR}_{1}$ and the KulbackLiebler divergence $\mathrm{KLD}_{1}$, nonlinear temporal correlations as described by the nonlinearity index $\xi$, and the asymmetric distribution of note intervals) describe different faces of the same concept. All of these properties indeed intervene in what could be called a "musical narrative," the flow of a composition. Furthermore, the finding that these three properties are statistically interrelated may be indicative of a deep relation between directionality, dissipation, and nonlinearity with pleasantness in music. Their role in the more general questions of perception, music appreciation, and cognition constitute open problems.

This work should be taken as a first step of a more in depth, inclusive research program. The three main elements of music are rhythm, melody, and harmony [52]. While here we have only addressed melody, we expect the integration of rhythm and harmony in our line of research to be enlightening. The update of our study in order to include the advent of atonaldodecaphonic, serial, stochastic, concrete, and spectral music, amongst others, is another fascinating open challenge.

\section{ACKNOWLEDGMENTS}

A.G.-E. thanks J. Plotkin for fruitful discussions and relevant comments in the development of this study. G.M.-M. thanks I. Darszon for sharing his musical insight, and R. Salgado and H. Larralde for valuable comments. G.M.-M. acknowledges the hospitality of the Ecole Normale Supérieure (Paris) during a sabbatical leave and the financial support from (PASPA/DGAPA/UNAM) during that period. L.L. acknowledges funding from EPSRC Early Career Fellowship No. EP/P01660X/1 and also acknowledges the hospitality of Ecole Normale Supérieure, where part of this research was conducted during a research stay.

A.G.-E., G.M.-M., and L.L. designed the study. A.G.-E. processed and curated the data. A.G.-E. and L.L. developed the research and implemented all methods. All authors discussed, interpreted results, and wrote the paper.

\section{APPENDIX A: CORRELATIONS AMONG DIFFERENT IRREVERSIBILITY RATIO ORDERS}

In the (a) and (b) panels of Fig. 14 we scatter-plot (in linear$\log$ scales, for the sake of illustration) the irreversibility ratios $\mathrm{IR}_{m}$ vs $\mathbf{I R}_{m-1}$ for every piece in the data set, and compute the Pearson correlation coefficient, finding $r=0.93$ for $\mathrm{IR}_{2}$ vs $\mathrm{IR}_{1}$ and $r=0.95$ for $\mathrm{IR}_{3}$ vs $\mathrm{IR}_{2}$, i.e., very strong correlation. 

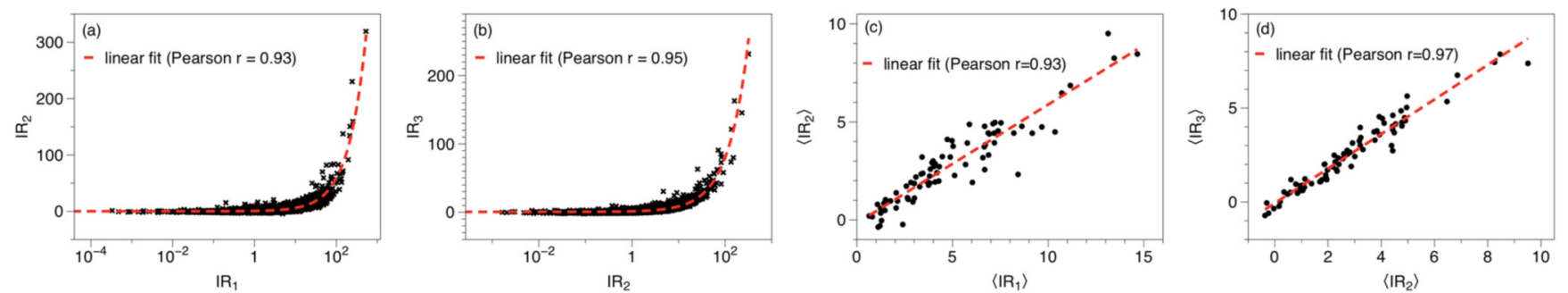

FIG. 14. Irreversibility ratios of different order are correlated. [Panels (a),(b)] Linear-log scatter plots of irreversibility ratios $\mathrm{IR}_{m} \mathrm{vs} \mathrm{IR}_{m-1}$ for each piece in the data set. The red dashed line is the best linear fit, concluding that all three orders are strongly correlated. [Panels (c),(d)] Linear scatter plots of composer-averaged irreversibility ratios. The strong correlation observed suggests that, for the set of signals analyzed in this work, $\mathrm{IR}_{1}$ is sufficient to characterize irreversibility and no higher-order quantifiers are needed.

In the (c) and (d) panels of the same figure, a similar analysis is performed (scatter-plots are in linear scales), but we average a single irreversibility ratio per composer (averaging over all the pieces for the same composer), and then correlate $\left\langle\mathrm{IR}_{m}\right\rangle$ $\mathrm{vs}\left\langle\mathrm{IR}_{m-1}\right\rangle$. Again we find very strong correlation $(r=0.92$ and 0.97 , respectively). Altogether, these results suggest that all three quantities are linearly correlated, meaning that (i) there are no high-order HVG-irreversibility structures in the set of musical compositions analyzed and (ii), accordingly, it is enough to set $m=1$ and safely focus on $\mathrm{IR}_{1}$ to fully account for irreversibility in this context.

\section{APPENDIX B: DFA AND MAGNITUDE DFA}

The detrended fluctuation analysis (DFA) method was developed by Peng et al. to compute the long range correlations in stationary and nonstationary time series [53]. A brief description of the method is as follows: for a given time series $x(i), i=1, \ldots, N$, the standard DFA method consists of the following steps. (1) The original signal is integrated $y(j)=$ $\sum_{i=1}^{j}[x(i)-\langle x\rangle]$, where $\langle x\rangle$ denotes its average value, (2) The integrated time series is then divided into nonoverlapping windows of size $s$. (3) Each data segment of length $s$ size is then fitted using a polynomial $y_{m}(j)$ of degree $m$. (4) Next, the root-mean-square fluctuation from the polynomial, $F(s)$, is calculated:

$$
F(s)=\sqrt{\frac{1}{N} \sum_{j=1}^{N}\left[y(j)-y_{m}(j)\right]^{2}} .
$$

The procedure is repeated by varying $s$ such that the fluctuation function is obtained in terms of the segment length, which represents the time scale where correlations might be present. When autocorrelations scale like a power law, the rms fluctuation function $F(s)$ behaves as $F(s) \sim s^{\alpha}$, where $\alpha$ is the Hurst exponent. A value of $\alpha>0.5$ indicates the presence of persistent correlations, e.g., $\alpha=1$ is the case for $1 / f$ noise. On the other hand, a value of $0<\alpha<0.5$ corresponds to anticorrelations and $\alpha=0.5$ to white noise. The magnitude detrended fluctuation analysis (MDFA) introduced by Ashkenazy et al. [42] is a method capable of detecting the presence of nonlinear correlations in a time series. This method can be summarized by the following recipe. (1) For a given time series $x(i)$ the increment series is defined as $\Delta x(i) \equiv x(i+1)-x(i)$. (2) The increment series is decomposed into a magnitude series and sign series: $\Delta x(i)=\operatorname{sgn}[\Delta x(i)]|\Delta x(i)| ;$ their respective means are subtracted to avoid artificial trends. (3) Because of the limitations of the DFA method for estimating $\alpha<0.5$ (anticorrelated series), the magnitude and sign series are integrated first to make sure they are positively correlated. (4) The DFA method is implemented on the integrated magnitude and sign series. (5) In order to obtain the respective scaling exponents, the function $F(s) / s$ is estimated; the $1 / s$ factor is to compensate the integration made before. If the data obey a scaling law, the fluctuation function should behave as $F(s) / s \sim s^{\alpha-1}$. It has been shown that the magnitude series $[|\Delta x(i)|]$ is the one that carries information regarding nonlinear correlations in the original time series [54].

To evaluate the evidence and amount of nonlinear correlations it is necessary to compare the MDFA results with the appropriate surrogate data results; these surrogates should preserve the linear correlations of the original time series but lack of any possible nonlinear correlations. We generate 20 surrogates for each piece with the iterative amplitude adjusted Fourier transform (IAAFT) algorithm [43,44] which seeks to preserve the power spectrum as well as the probability distribution function (PDF) of the original signal; the latter is its distinctive feature, achieved through the iterations. In music scores, it has been shown that a unique scaling in the $\log [F(s)]$ function is not always present; some variations with different scaling regimes and even the absence of scaling have been identified previously [20]. Diverse scaling profiles correspond to different types of autocorrelations (short and long range) that are sensitive to the structure of the musical piece. Figure 15 shows some examples of different profiles in the $\log [F(s)]$ function; panels (a) are the DFA results for the original piece (red crosses). The shaded area indicates the region which within DFA results for surrogates series are contained. The straight lines are linear regressions (vertically translated for visualization purposes) of the crosses within different scaling regimes. Notice that the results between the original and the surrogates in the three different examples are statistically indistinguishable; dots fall within the gray region. This fact reinforces the choice of the surrogates as null model. The above is not the case for the results of the DFA of the magnitude series shown in panel (b), where there is a difference between the original data and the surrogates; many crosses are located outside the gray region, which is indicative of the presence of nonlinear correlations in the original signal. (See also Fig. 16.) 

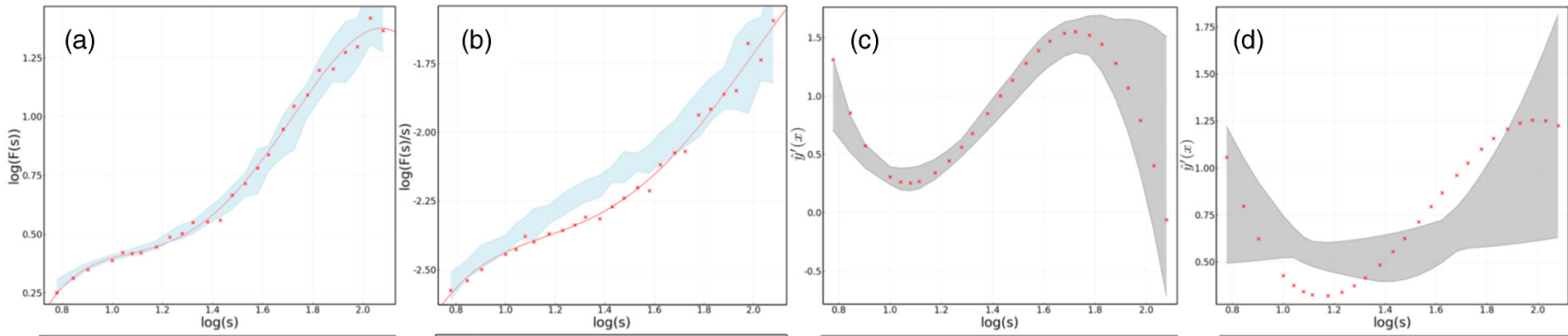

$\frac{5}{7}$
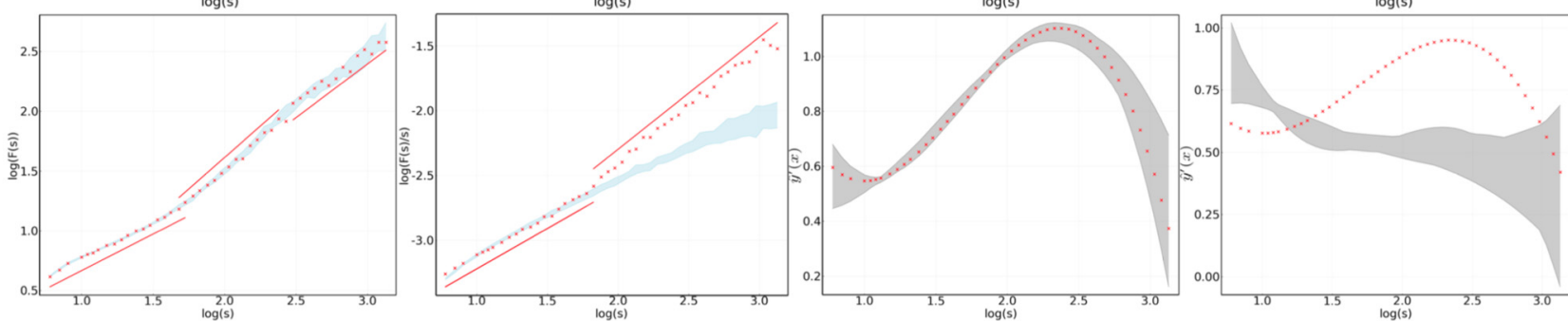

怘
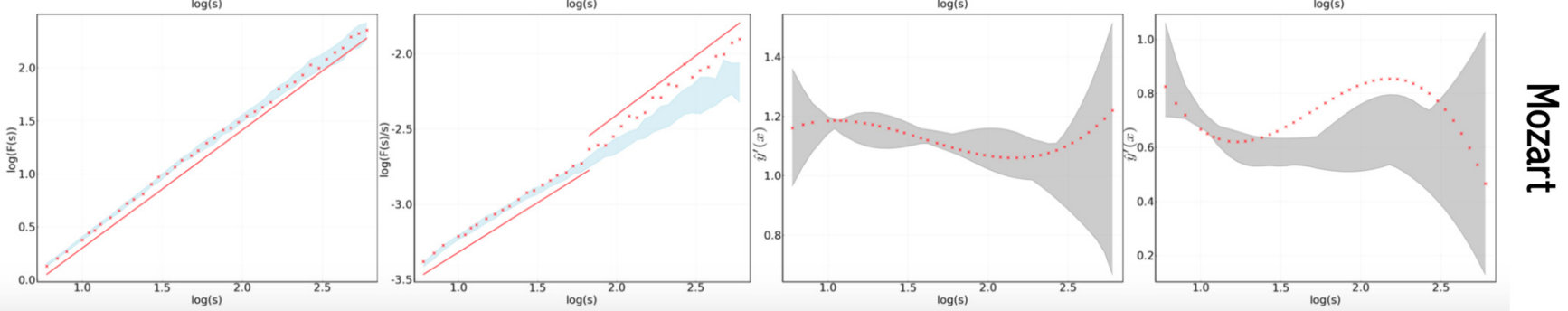

FIG. 15. DFA and MDFA calculations. Three different examples (pieces) from the corpus, from top to bottom: Liszt (excerpt from Christmas tree suite), Bartok (excerpt from mikrokosmos), and Mozart (violin sonata no. 21). The panel columns are (a) DFA computation (red crosses) with 20 surrogates represented by the shaded area. (b) MDFA computation performed as in the DFA case. (c) Slope valuespolynomial fit derivatives-for the DFA computation. (d) Slope values-polinomial fit derivatives-for the MDFA computation. Continuous curves are the polynomial fits of red crosses and straight lines come from linear regression, of red crosses within scaling ranges, vertically translated for visualization purposes.

To be able to quantify this difference in the amount of nonlinearity, we define a nonlinearity index $\xi$, given by comparing the scaling behavior of the original data and its surrogates. Usually when there is only one scaling region, the comparison is given in terms of the slope of the original data and the slopes of the null model [42]. In our case, instead of having a single scaling we have regions with different scalings. Therefore, in order to compare both scaling behaviors we first fit a polynomial $\hat{y}$ to the original MDFA data and also to each of its surrogates $\left(\hat{y}_{s}\right)$, evaluate the first derivative of $\hat{y}$ (slopes) at each point $[\log (s)]$ in the MDFA, and compute the index $\xi$ as follows:

$$
\xi=\frac{1}{N_{w s}} \sum_{i=1}^{N_{w s}} \frac{\left|\hat{y}^{\prime}\left(x_{i}\right)-\left\langle\hat{y}_{s}^{\prime}\left(x_{i}\right)\right\rangle_{\mathrm{sur}}\right|}{\sigma\left(\hat{y}_{s}^{\prime}\left(x_{i}\right)\right)_{\mathrm{sur}}}
$$

where $N_{w s}$ is the total number of window sizes, $\hat{y}^{\prime}\left(x_{i}\right)$ is the slope the polynomial evaluated at the $i$ th window size $\left[x_{i}=\log \left(s_{i}\right)\right]$, and $\left\langle\hat{y}_{s}^{\prime}\left(x_{i}\right)\right\rangle_{\text {sur }}$ and $\sigma\left(\hat{y}_{s}^{\prime}\left(x_{i}\right)\right)_{\text {sur }}$ represent the mean and variance of the slopes in the ensemble of surrogates at the $i$ th window size, respectively. By construction, $\xi \leqslant 1$ would indicate that the signal only evidences (at most) linear
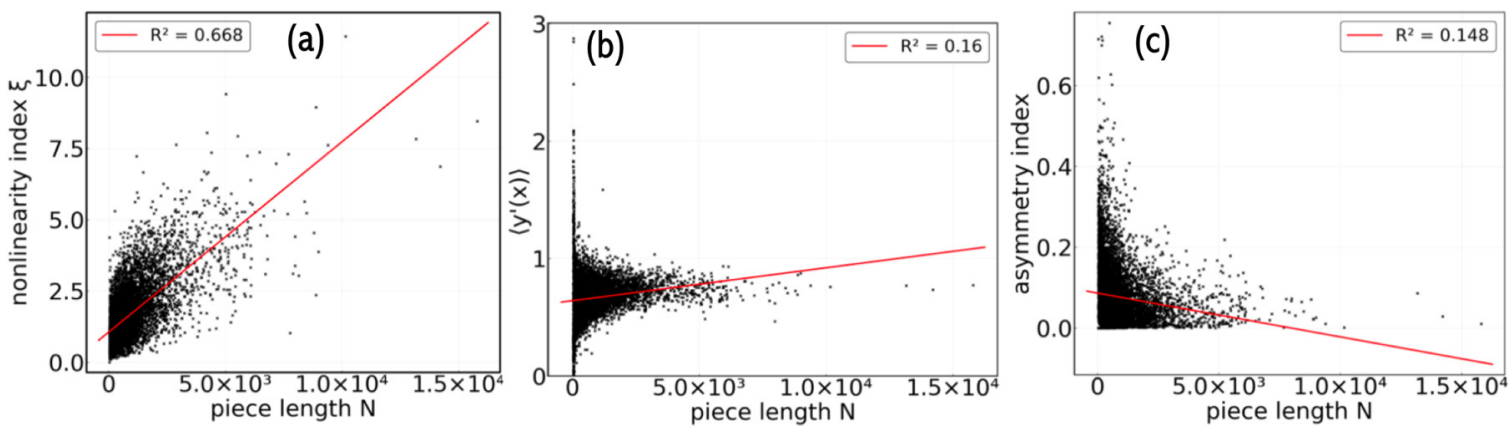

FIG. 16. Effect of piece length on the nonlinearity index $\xi$ [panel (a)], mean local slope $\left\langle\hat{y}^{\prime}(x)\right\rangle$ [panel (b)], and asymmetry $D_{\uparrow \downarrow}$ [panel (c)]. 

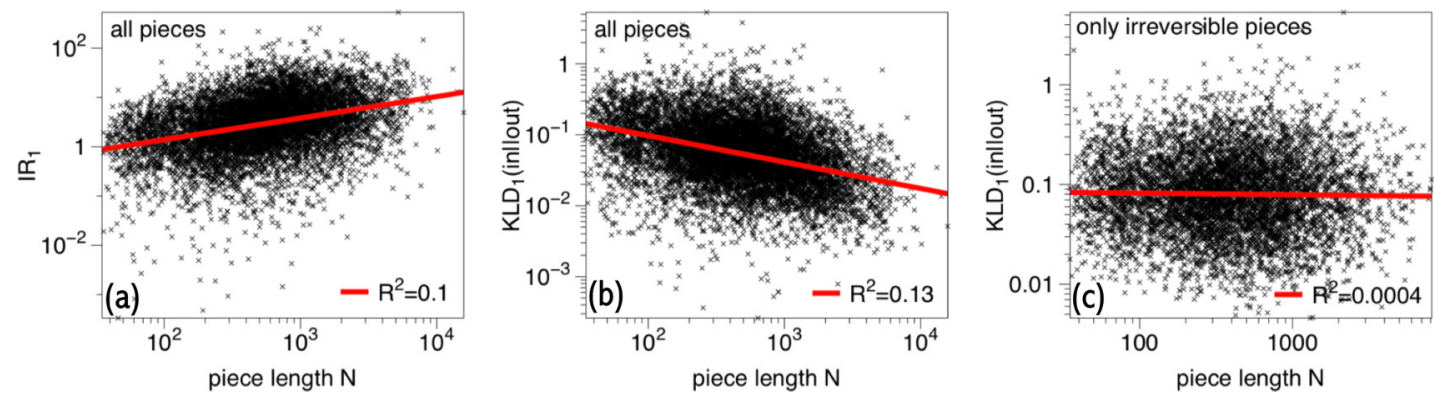

FIG. 17. Effect of piece length on $\mathrm{IR}_{1}$ and $\mathrm{KLD}_{1}(\mathrm{in}||$ out$)$. As shown by $R^{2}$, there are subtle correlations of the irreversibility ratio and irreversibility value with piece length [panels (a),(b)], and this correlation is removed [panel (c)] if we only consider the irreversibility value $\mathrm{KLD}_{1}(\mathrm{in}||$ out$)$, the pieces of which have been previously certified to be irreversible $\left(\mathrm{IR}_{1}>1\right)$.

correlations, whereas if $\xi>1$ the signal has correlations of nonlinear nature (not reflected in the power spectrum), and the larger $\xi$ the stronger.

To exemplify some values of the index, the results for $\xi$ in the three different examples given in Fig. 15 are, for Liszt, $\xi_{D F A}=0.79$ and $\xi_{M D F A}=1.73$, for Bartok, $\xi_{D F A}=0.42$ and $\xi_{M D F A}=7.4$, and in the case of Mozart $\xi_{D F A}=0.53$ and $\xi_{M D F A}=2.43$. These results show how the index $\xi$ is able to identify and quantify the difference in the magnitude DFA results; meanwhile, in the case of the DFA, since all indexes are below 1, surrogates and the original signal are statistically indistinguishable. In the manuscript we use this index only for the nonlinearity in the MDFA computation. It is important to remark that in the case of the Mozart example, unique scaling in the DFA does not necessary imply absence of nonlinearity.

\section{APPENDIX C: DEPENDENCIES OF IR 1 AND KLD $($ in||out) WITH SERIES SIZE}

According to the theoretical analysis conducted in Fig. 6, let us assume that a time series of size $N$ is generated by a certain dynamical process. Then we have the following.

(i) If the process is reversible, then $\mathrm{IR}_{1}$ is systematically below 1 and is insensitive to series size $N$ and, on the other hand, $\mathrm{KLD}_{1}$ (in||out) decreases with series size.

(ii) If the process is irreversible, then $\mathrm{KLD}_{1}$ (in||out) is reasonably stable and insensitive to series size $N$, whereas $\mathrm{IR}_{1}$ is expected to grow with series size.

Let us consider now the musical pieces. Each of them is composed by different composers (so a priori by a possibly different "dynamical process"), and each of them has different length, spanning from dozens to thousands of notes. Comparison across composers and pieces is therefore difficult. In Fig. 17 we depict the values of $\mathrm{IR}_{1}$ and $\mathrm{KLD}_{1}$ (in||out) as a function of the piece length $N$ for all pieces in the data set (left and middle panels) and only for those pieces that have been certified as irreversible, i.e., those for which $\mathrm{IR}_{1}>1$. Panels are in log-log to have a better visualization of all the points. We can see in the left panel a small increasing trend: this is indeed related to all those pieces which are irreversible, whose irreversibility ratio $\mathrm{IR}_{1}$ tends to be larger for larger time series. Similarly, in the middle panel we can appreciate a subtle decreasing trend: this is indeed related to all those pieces which are reversible, whose reversibility value $\mathrm{KLD}_{1}$ (in||out) tends to be smaller for larger time series. In the right panel there is no trend with series length $N$, which is indicative of the size independence of the irreversibility value $\mathrm{KLD}_{1}$ (in||out).
[1] R. F. Voss and J. Clarke, '1/fnoise' in music and speech, Nature 258, 317 (1975).

[2] R. F. Voss and J. Clarke, " $1 / f$ noise" in music: Music from $1 / f$ noise, J. Acoust. Soc. Am. 63, 258 (1978).

[3] D. J. Levitin, P. Chordia, and V. Menon, Musical rhythm spectra from Bach to Joplin obey a $1 / f$ power law, Proc. Natl. Acad. Sci. USA 109, 3716 (2012).

[4] D. Wu, K. Kendrick, D. Levitin, C. Li, and D. Yao, Bach is the father of harmony: revealed by a $1 / \mathrm{f}$ fluctuation analysis across musical genres, PLoS ONE 10, e0142431 (2015).

[5] H. Hennig, Synchronization in human musical rhythms and mutually interacting complex systems, Proc. Natl. Acad. Sci. USA 111, 12974 (2014).

[6] G. Gündüz and U. Gündüz, The mathematical analysis of the structure of some songs, Physica A: Stat. Mech. Appl. 357, 565 (2005).
[7] L. Telesca and M. Lovallo, Analysis of temporal fluctuations in Bach's sinfonias, Physica A: Stat. Mech. Appl. 391, 3247 (2012).

[8] L. Dagdug, J. Alvarez-Ramirez, C. Lopez, R. Moreno, and E. Hernandez-Lemuz, Correlations in a Mozart's music score (K-73x) with palindromic and upside-down structure, Physica A: Stat. Mech. Appl. 383, 570 (2007).

[9] G. R. Jafari, P. Pedram, and L. Hedayatifar, Long-range correlation and multifractality in Bach's inventions pitches, J. Stat. Mech. (2007) P04012.

[10] H. D. Jennings, P. Ch. Ivanov, Ade. M. Martins, P. C. da Silva, and G. M. Viswanathan, Variance fluctuations in nonstationary time series: a comparative study of music genres, Physica A: Stat. Mech. Appl. 336, 585 (2004).

[11] H. Hennig, R. Fleischmann, A. Fredebohm, Y. Hagmayer, J. Nagler, A. Witt, F. J. Theis, and T. Geisel, The nature and 
perception of fluctuations in human musical rhythms, PLoS ONE 6, e26457 (2011).

[12] L. Liu, J. Wei, H. Zhang, J. Xin, and J. Huang, A statistical physics view of pitch fluctuations in the classical music from Bach to Chopin: evidence for scaling, PLoS ONE 8, e58710 (2013).

[13] X. F. Liu, C. K. Tse, and M. Small, Complex network structure of musical compositions: algorithmic generation of appealing music, Physica A: Stat. Mech. Appl. 389, 126 (2010).

[14] M. Schroeder, Fractals, Chaos, Power Laws: Minutes from an Infinite Paradise (Dover Publications, Mineola, NY, 1991).

[15] B. West and M. Shlesinger, The noise in natural phenomena, Am. Sci. 78, 1 (1990).

[16] D. L. Gilden, T. Thornton, and M. W. Mallon, $1 / f$ noise in human cognition, Science 267, 1837 (1995).

[17] W. H. Press, Flicker noises in astronomy and elsewhere, Comments Astrophys. 7, 103 (1978).

[18] P. Bak, How Nature Works: The Science of Self-organized Criticality (Springer, Berlin, 1996).

[19] S. Kauffman, At Home in the Universe: The Search for Laws of Self-organization and Complexity (Oxford University Press, Oxford, 1995).

[20] A. Gonzalez-Espinoza, H. Larralde, G. Martinez-Mekler, and M. Muller, Multiple scaling behavior and nonlinear traits in music scores, R. Soc. Open Sci. 4, 171282 (2017).

[21] C. F. Hastey, On the problem of succession and continuity in twenthieth-century music, Music theory spectrum 8, 58 (1986).

[22] P. G. Vos and J. M. Troost, Ascending and descending melodic intervals: statistical findings and their perceptual relevance, Music Percept.: Interdisciplin. J. 6, 383 (1989).

[23] M. J. Grant, Serial Music, Serial Aestetics (Cambridge University Press, Cambridge, UK, 2001).

[24] G. Weiss, Time-reversibility of linear stochastic processes, J. Appl. Prob. 12, 831 (1975).

[25] E. Roldán and J. M. R. Parrondo, Estimating Dissipation from Single Stationary Trajectories, Phys. Rev. Lett. 105, 150607 (2010).

[26] E. Roldán, J. Barral, P. Martin, J. M. R. Parrondo, and F. Julicher, Arrow of time in active fluctuations, arXiv:1803.04743.

[27] E. Roldán, I. Neri, M. Dorpinghaus, H. Meyr, and F. Julicher, Decision Making in the Arrow of Time, Phys. Rev. Lett. 115, 250602 (2015).

[28] C. S. Daw, C. E. A. Finney, and M. B. Kennel, Symbolic approach for measuring temporal "irreversibility", Phys. Rev. E 62, 1912 (2000).

[29] L. Lacasa, A. Nuñez, E. Roldan, J. M. R. Parrondo, and B. Luque, Time series irreversibility: a visibility graph approach, Eur. Phys. J. B 85, 217 (2012).

[30] L. Lacasa and R. Flanagan, Time reversibility from visibility graphs of non-stationary processes, Phys. Rev. E 92, 022817 (2015).

[31] Kunst der fugue: The largest resource of classical music in midi files, http://www.kunstderfuge.com/.

[32] Midicsv: convert midi file to and from csv, https://www. fourmilab.ch/webtools/midicsv/.

[33] A. González-Espinoza, https://github.com/spiralizing/MidiTS. jl.

[34] E. Roldan and J. M. R. Parrondo, Entropy production and Kullback-Leibler divergence between stationary tra- jectories of discrete systems, Phys. Rev. E 85, 031129 (2012).

[35] L. Lacasa, B. Luque, F. Ballesteros, J. Luque, and J. C. Nuño, From time series to complex networks: The visibility graph, Proc. Natl. Acad. Sci. USA 105, 4972 (2008).

[36] B. Luque, L. Lacasa, F. Ballesteros, and J. Luque, Horizontal visibility graphs: Exact results for random time series, Phys. Rev. E 80, 046103 (2009).

[37] M. Newman, The structure and function of complex networks, SIAM Rev. 45, 167 (2003).

[38] Z.-K. Gao, M. Small, and J. Kurths, Complex network analysis of time series, Europhys. Lett. 116, 50001 (2017).

[39] Y. Zou, R. V. Donner, N. Marwan, J. F. Donges, and J. Kurths, Complex network approaches to nonlinear time series analysis, Phys. Rep. 787, 1 (2019).

[40] R. Flanagan and L. Lacasa, Irreversibility of financial time series: a graph-theoretical approach, Phys. Lett. A 380, 1689 (2016).

[41] L. Lacasa, On the degree distribution of horizontal visibility graphs associated with Markov processes and dynamical systems: diagrammatic and variational approaches, Nonlinearity 27, 2063 (2014).

[42] Y. Ashkenazy, S. Havlin, P. Ch. Ivanov, C.-K. Peng, V. SchulteFrohlinde, and H. E. Stanley, Magnitude and sign scaling in power-law correlated time series, Physica A: Stat. Mech. Appl. 323, 19 (2003).

[43] Timeseriessurrogates: A julia library for generating surrogate data: https://github.com/kahaaga/timeseriessurrogates.j1/.

[44] T. Schreiber and A. Schmitz, Surrogate time series, Physica D: Nonlin. Phenom. 142, 346 (2000).

[45] J. Theiler, S. Eubank, A. Longtin, B. Galdrikian, and J. D. Farmer, Testing for nonlinearity in time series: the method of surrogate data, Physica D: Nonlin. Phenom. 58, 77 (1992).

[46] M. Beltrán del Río, G. Cocho, and G. G. Naumis, Universality in the tail of musical note rank distribution, Physica A 387, 5552 (2008).

[47] G. Martínez-Mekler, R. Alvarez Martínez, M. Beltrán del Río, R. Mansilla, P. Miramontes, and G. Cocho, Universality of rank-ordering distributions in the arts and sciences, PLoS ONE 4, e4791 (2009).

[48] J. Useche and R. Hurtado, Melodies as maximally disordered systems under macroscopic constraints with musical meaning, Entropy 21, 532 (2019).

[49] D. Zanette, Zipf's law and the creation of musical context, Music. Sci. 10, 3 (2006).

[50] G. Niklasson and M. Niklasson, Non-Gaussian distributions of melodic intervals in music: The Lévy-stable approximation, Europhys. Lett. Lett. J. Explor. Front. Phys. 112, 40003 (2015).

[51] I. Xenakis, Formalized Music: Thought in Mathematics and Composition (Pendragon Press, Stuyvesant, NY, 1992).

[52] D. D. Boyden, An Introduction to Music, 2nd ed. (Faber and Faber Ltd., London, 1971).

[53] C.-K. Peng, S. C. Buldyrev, S. Havlin, M. Simons, H. E. Stanley, and A. L. Goldberger, Mosaic organization of DNA nucleotides, Phys. Rev. E 49, 1685 (1994).

[54] Y. Ashkenazy, P. Ch. Ivanov, S. Havlin, C.-K. Peng, A. L. Goldberger, and H. E. Stanley, Magnitude and Sign Correlations in Heartbeat Fluctuations, Phys. Rev. Lett. 86, 1900 (2001). 